Sociologie et sociétés

\title{
Le mariage, quelle affaire !
}

Encadrement social et privatisation de l'entrée en union en milieu rural malien

Marriage, What a Business !

\author{
Social Framework and Prevention of Union in Rural Areas of \\ Mali
}

\section{Véronique Hertrich}

Volume 39, numéro 2, automne 2007

Sociétés africaines en mutation : entre individualisme et communautarisme

Mutating African Societies: Between Individualism and Communitarism

URI : https://id.erudit.org/iderudit/019087ar

DOI : https://doi.org/10.7202/019087ar

Aller au sommaire du numéro

\section{Éditeur(s)}

Les Presses de l'Université de Montréal

ISSN

0038-030X (imprimé)

1492-1375 (numérique)

Découvrir la revue

Citer cet article

Hertrich, V. (2007). Le mariage, quelle affaire ! Encadrement social et privatisation de l'entrée en union en milieu rural malien. Sociologie et sociétés, 39(2), 119-150. https://doi.org/10.7202/019087ar

\section{Résumé de l'article}

L'article examine l'individualisation des formes d'entrée en union dans une population rurale du Mali, à partir d'une enquête biographique détaillant les procédures matrimoniales (initiative, prestations, médiatisation et formalisation) et à partir d'entretiens qualitatifs. Il relativise l'idée d'un mouvement continu et homogène bâti sur la contestation individuelle des contrôles familiaux. Le mariage a d'abord évolué par réajustements successifs en intégrant les attentes masculines au dispositif formel géré par les aînés. Il connaît une évolution récente plus brutale, marquée par le recul de la formalisation des unions et le désengagement des responsables familiaux de la prospection matrimoniale. Ce tournant est associé à l'essor des migrations féminines qui met à mal le dispositif institutionnel d'encadrement des jeunes. L'individualisation se double d'un mouvement de privatisation des affaires matrimoniales. Le mariage reste un élément important du jeu collectif, mais avec un glissement de paradigme : il était une composante du contrôle intergénérationnel, il devient aujourd'hui un instrument de différenciation sociale. 


\section{Le mariage, quelle affaire!}

Encadrement social et privatisation de l'entrée en union en milieu rural malien

\section{VÉRONIQUE HERTRICH}

Institut national d'études démographiques (INED)

133, boulevard Davout

75980 Paris cedex 20, France

Courriel: hertrich@ined.fr

$\mathrm{D}$

ANS la Plupart des sociétés D’Afrique subsaharienne, le mariage correspond à un temps particulièrement fort de la vie sociale, autour duquel se cristallisent attentes individuelles et exigences collectives ${ }^{1}$. Pour l'individu, il est une étape décisive du cycle de vie, significative d'une évolution statutaire, s'accompagnant de nouvelles responsabilités et de nouveaux droits, en particulier l'accès à une vie sexuelle, conjugale et reproductive socialement reconnue. Pour la collectivité, le mariage est une composante essentielle de la reproduction démographique et sociale: il définit des droits sur la descendance des femmes et organise ainsi la filiation; il alimente aussi, par le jeu des alliances, les rapports politiques et sociaux entre les groupes. Enfin, les arrangements matrimoniaux mettent en scène les formes de domination et d'inégalité qui structurent les rapports sociaux, notamment entre les sexes et entre les générations. Ils en sont à la fois des instruments et des révélateurs. L'affaire matrimoniale se situe de fait à l'intersection de logiques variables: celles de la communauté élargie, avec ses valeurs et ses institutions, celles des individus qui se marient, avec leurs attentes personnelles,

1. Pour une vue synthétique sur les enjeux associés au mariage d'après les différentes théories, voir par exemple Mair (1971), Burnham (1987), Hertrich et Locoh (1999), Antoine (2002). 
mais aussi celles d'unités intermédiaires et d'individus autres, qui ont leurs propres intérêts.

Comment ce conflit d'intérêts potentiel est-il géré?

La complexité des procédures matrimoniales observée en Afrique subsaharienne peut être considérée comme une réponse institutionnelle destinée à désactiver ces sources d'antagonisme et à maintenir un contrôle, à l'échelle communautaire, sur la formation des couples. La littérature ethnographique témoigne de l'énergie déployée par les sociétés pour imaginer, avec profusion de détails, la formalisation de l'appariement entre les sexes. Une telle diversité dans l'organisation des procédures matrimoniales permet raisonnablement d'avancer que leur principal point commun réside dans leur complexité. On peut cependant identifier des traits récurrents, prenant des déclinaisons variables: l'inscription dans la durée de la procédure matrimoniale, la circulation de ressources matérielles ou symboliques, l'aspect protocolaire des échanges entre les deux parties de l'alliance, la participation d'intervenants extérieurs aux familles, la tenue de cérémonies publiques. L'inclusion de dimensions multiples dans le montage matrimonial est certainement un frein essentiel à son appropriation au bénéfice d'intérêts particuliers. Pour les intéressés, la mise en œuvre du mariage s'avère inaccessible car trop compliquée. Quant aux responsables familiaux, en charge de l'organisation matrimoniale, leur marge de manœuvre est limitée par l'encadrement social de la pratique (codification, médiation communautaire...).

La nature de ces agencements, associant des éléments et des registres variés, est certainement une explication à la persistance des formes d'encadrement de l'entrée en union, observée bien souvent en milieu rural africain en dépit d'une participation personnelle croissante des intéressés à la décision matrimoniale ${ }^{2}$. En jonglant avec les différentes composantes du jeu matrimonial, il est possible d'en abandonner certaines, d'en redéfinir d'autres, de se positionner sur des registres variables et ainsi de maintenir un cadre formel susceptible d'intégrer de nouvelles orientations ou de concilier des exigences différentes.

Cet article examine l'organisation et l'évolution de ces arrangements matrimoniaux à partir d'une recherche approfondie à petite échelle au Sud-Est du Mali. L'accent est mis sur la dynamique de ces pratiques, d'un double point de vue: celui de la souplesse du système matrimonial et de sa capacité à concilier attentes individuelles et collectives, celui des transformations en cours et de leur signification dans l'organisation des rapports entre sexes et entre générations.

Le propos est organisé en trois principales parties, précédées d'une brève présentation de la population et des données étudiées. La première partie porte sur le contrôle social de l'entrée en union dans cette population : comment ce contrôle se réalise-t-il? pourquoi est-il accepté par les jeunes? quelles sont les conditions de son efficacité? La deuxième partie examine, statistiques à l'appui, l'évolution des procédures matrimo-

2. Voir par exemple les études réunies par Parkin et Nyamwaya (1987), Bledsoe et Pison (1994), ou citées par Lloyd (2005). 
niales au cours des dernières décennies et leur incidence sur les rapports intergénérationnels. Enfin, pour conclure, une dernière partie ouvre la discussion sur les ressorts et la portée des transformations récentes, en distinguant la question de l'individualisation de celle de la privatisation des affaires matrimoniales.

\section{CADRAGE: LA POPULATION, LES DONNÉES}

La population étudiée se situe au Sud-Est du Mali, dans l'aire ethnique des Bwa (au singulier, boo), à 450 kilomètres environ de Bamako. Un système d'observation ${ }^{3}$ conciliant différents types d'opérations (recensement, enquêtes, généalogie...) y a été mis au point à la fin des années 1980, et fait depuis l'objet d'une actualisation régulière, tous les 5 ans environ, la dernière datant de 2004.

\section{Les données}

J'utilise principalement ici les données de l'enquête biographique, réalisée exhaustivement dans deux villages, Sirao (Hanfwa'ui) et Kwara (1 600 habitants en 2004). Cette enquête collecte les histoires matrimoniale, génésique, migratoire et religieuse de l'ensemble des résidants — hommes et femmes — et d'une partie des émigrés. La biographie matrimoniale y est enregistrée de façon particulièrement détaillée pour permettre d'analyser l'évolution des contrôles communautaires sur la formation des couples. Des questions sont ainsi posées sur les différentes étapes des procédures matrimoniales (initiative, prestations, médiation, durée de la procédure, cérémonies...), y compris les procédures qui ont échoué avant la conclusion de l'union. L'annexe fournit des informations techniques sur ces données.

À titre secondaire, j'utilise les entretiens réalisés en 2002 dans le cadre d'une enquête qualitative sur l'expérience des rapports entre sexes et générations au cours des différents âges de la vie (65 hommes et femmes interrogés), dont je citerai quelques extraits en guise d'exemple.

Enfin, l'observation permise par une présence répétée sur le terrain depuis une vingtaine d'années et les échanges informels qu'elle induit ont alimenté les réflexions sur les ressorts des évolutions en cours et le repérage des changements les plus récents.

\section{Contexte socioéconomique}

La population vit de l'agriculture, principalement vivrière, réalisée dans le cadre d'un mode de production familial; le commerce et les cultures de rente sont peu développés. Trait particulier dans un Mali majoritairement musulman, les Bwa sont restés hermétiques à l'islam mais se sont partiellement ouverts au christianisme, tout en maintenant les cultes communautaires traditionnels.

Du point de vue démographique (Hertrich et Lesclingand, 2003), la région est marquée par une forte croissance naturelle (2,5\% à $3 \%$ par an), en grande partie

3. Pour une présentation plus détaillée du système de collecte et de la population étudiée, voir Hertrich (1996) et Hertrich et Lesclingand (2003). 
corrigée par la dynamique migratoire. La mortalité a connu une baisse significative depuis les années 1950 mais touche encore 1 enfant sur 6 avant 5 ans. La fécondité se maintient à un niveau très élevé, de l'ordre de 8 enfants par femme.

Les années 1990, marquées à l'échelle du Mali par l'avènement de la démocratie (1991) et l'adoption d'une politique de décentralisation multisectorielle (1993) (Keïa et Konaté, 2003), correspondent aussi, à l'échelle locale, à une période d'ouverture et de début d'engagement dans des projets politiques et des programmes de développement. Il s'agit là d'un tournant de la part d'une population qui avait toujours marqué ses distances avec les structures nationales (Diarra, 1994). L'établissement d'écoles communautaires, gérées et financées par les villageois, montre ainsi l'investissement récent des communautés dans le projet scolaire. Jusqu'alors, le niveau de scolarisation avait été particulièrement faible: dans les deux villages étudiés, ce sont à peine $15 \%$ des enquêtés des générations 1950-1975 qui ont été à l'école et moins de $5 \%$ qui ont suivi une scolarisation primaire complète. Parmi les générations nées à la fin des années 1980, ce sont plus de $40 \%$ des enfants qui ont été envoyés à l'école, $60 \%$ dans le village ayant sa propre école. Les années 1990 se caractérisent également par la généralisation des migrations de travail des jeunes. Le phénomène avait débuté chez les hommes dès la fin des années 1960, mais ce n'est que depuis une quinzaine d'années qu'il a commencé à s'imposer également chez les jeunes femmes (Lesclingand, 2004a, 2004b). Dès l'âge de 12 ou 13 ans, ces dernières partent travailler en ville comme domestiques ("petites bonnes»), tandis que les jeunes hommes vont habituellement d'abord s'employer en milieu rural (gardiennage de troupeaux pour le compte d'éleveurs Peul) avant d'aller en ville. Cette pratique est devenue aujourd'hui une composante quasi incontournable du passage à l'âge adulte pour les deux sexes: 95 \% des jeunes nés au début des années 1980 ont réalisé au moins une migration de travail avant leur vingtième anniversaire (Hertrich et Lesclingand, 2003, 2007). L'expérience de la période adolescente s'en trouve modifiée, avec un élargissement de l'espace de vie au delà de leur aire ethnique pour $80 \%$ des jeunes (contre à peine $15 \%$ au sein des générations nées avant 1950). La vie communautaire s'en trouve également changée, avec une présence de moins en moins visible des jeunes au village.

On retrouvera dans les tendances du mariage ce point d'inflexion des années 1990.

\section{Famille et village}

L'encadrement social des individus s'organise principalement par l'intermédiaire de trois groupes de rattachement: le patrilignage, le groupe domestique et le village. Le patrilignage est le référent dans le domaine sociopolitique (droits fonciers, prérogatives politiques et rituelles au village) et matrimonial (exogamie, décisions matrimoniales). Le lignage, selon sa taille, compte un nombre variable de groupes domestiques $(z \hat{u})$ ou exploitations agricoles, de structure souvent complexe ${ }^{4}$. Le village occupe une

4. Plus de la moitié de la population vit dans une configuration familiale polynucléaire et appartient à une unité économique comptant 10 membres au moins. Ces caractéristiques ont peu évolué au cours des 30 dernières années (Lesclingand et Hertrich, 2006). 
place privilégiée dans la vie sociale des Bwa (Capron, 1973, 1988a, 1988b; SavonnetGuyot, 1986). Cette orientation se perçoit dans l'organisation sociopolitique villageoise de structure plurilignagère, mais aussi plus concrètement dans la structure spatiale du village et dans l'importance des pratiques communautaires, notamment festives. À la différence d'autres populations de la région, chez les Bwa, les unités familiales ne s'inscrivent pas dans un espace résidentiel délimité (concession). Au contraire, les membres d'un groupe domestique se répartissent généralement dans différentes cases, fondues dans la masse villageoise et s'ouvrant sur la rue. C'est au sein de l'espace public que prennent place l'essentiel des activités quotidiennes. La vie communautaire, entretenue par des relations de voisinage omniprésentes, est par ailleurs valorisée par de nombreuses manifestations festives. Les cérémonies rituelles traditionnelles ou chrétiennes, les cérémonies familiales (mariage, funérailles), les parties de travail réalisées par un groupe de jeunes pour le compte d'un exploitant, dans le cadre des prestations matrimoniales ou encore dans un cadre associatif, sont autant d'occasions festives auxquelles s'associent les villageois. À ces manifestations occasionnelles s'ajoute l'institution dans chaque village d'une journée hebdomadaire réservée à la consommation de boisson (bière de mil) au cours de laquelle les tâches quotidiennes sont suspendues au profit de réjouissances collectives. Cette sociabilité villageoise forge un sentiment d'appartenance et un attachement fort des individus à leur village. Dans le même temps elle assure un encadrement communautaire permanent et, par là même, l'exercice d'un contrôle social diffus, qui entrave le développement de la sphère privée. Cette vie communautaire est facteur d'homogénéité socioéconomique: les surplus économiques sont dépensés les jours de boisson et de fête, décourageant accumulation et investissement. Elle conditionne aussi les formes du changement social: il est difficile d'innover, mais quand un nouveau comportement est introduit, il se diffuse rapidement.

\section{L'ENCADREMENT DE L'ENTRÉE EN UNION}

Il est de la responsabilité des lignages d'organiser l'entrée en union de leurs membres. On cherche des épouses aux hommes et on accorde les filles en mariage. Les responsables familiaux disposent d'une autorité et d'une compétence dans la gestion matrimoniale qui leur sont largement reconnues. Jusque récemment la plupart des mariages entre célibataires faisaient suite à un accord formalisé entre les deux familles ${ }^{5}$. Dans les années 1970, c'était encore le cas de plus de 8 mariages sur 10. Comment ce système fonctionne-t-il? Quels sont les fondements de sa robustesse? de son acceptation par les jeunes? Les réponses sont à chercher dans les cadres matrimoniaux, mais aussi dans l'organisation de la socialisation juvénile et des rapports intergénérationnels. D’une certaine manière, tous ces arrangements convergeaient pour désintéresser les jeunes de la question de leur mariage: des procédures trop compliquées et trop incertaines pour tenter un jeune inexpérimenté; un temps de jeunesse autrement plus intéressant

5. Seuls les mariages avec une jeune femme célibataire font l'objet d'une formalisation, les remariages féminins étant généralement très libres. 
à vivre; enfin, un système efficace assurant sans inégalités criantes l'entrée en union de chacun dans des délais raisonnables.

\section{La jeunesse, un temps d'insouciance}

Les Bwa disposent d'un terme, yaromu ${ }^{6}$, pour parler du temps de jeunesse avant le mariage. Il est compris comme une période d'exception, où l'on a acquis la maturité physique d'un adulte ${ }^{7}$ tout en étant encore épargné par les responsabilités.

L'entrée dans la jeunesse est associée à un nouveau style de vie, dominé par les relations entre pairs. Garçons et filles quittent généralement la case de leurs parents pour dormir entre amis, tout en retrouvant leur famille la journée pour les repas et les travaux. Les garçons se regroupent à 3 ou 4, dans des cases délaissées du village, tandis que les jeunes filles restent sous la responsabilité d'un adulte, généralement une vieille femme.

La jeunesse est vécue comme une période d'insouciance, de distractions, placée sous le signe de la sociabilité et de la plaisanterie. Pendant la saison sèche, les jeunes hommes se déplacent entre amis pour des parties de «causerie» auprès des filles. Certaines nuits de pleine lune, les filles organisent des séances de danse propres à leur âge (nabwo), tandis que les garçons, autrefois, s'occupaient à des parties de lutte sur la même esplanade. Pendant la saison agricole, les jeunes montent des associations de travail, destinées à financer une fête après les récoltes, ou se mobilisent afin d'assumer les prestations agricoles pour le mariage de l'un des leurs. Les communautés chrétiennes sont souvent rejointes par les adolescents (et abandonnées quelques années plus tard) en tant qu'elles offrent un cadre de sociabilité et de divertissement supplémentaire. Les relations entre pairs, du ressort de l'amitié et de l'entraide, laissent aussi une place à la compétitivité et à l'affirmation de soi. Dans l'exercice sportif (danses, parties de travail, lutte), les jeunes, en particulier les garçons, se mesurent les uns aux autres et trouvent à s'exprimer collectivement ou individuellement par la valorisation de la force physique.

Ces scènes publiques sont aussi des lieux où les jeunes des deux sexes vont pouvoir se jauger, s'approcher, «se courtiser». Les jeunes filles font les éloges ou décrient les performances masculines; les jeunes hommes observent et taquinent les jeunes filles. Il y a place pour les affinités personnelles, dans le cadre de la jeunesse. Un jeune parlera de sa hazunu ( «sa fille») pour désigner son élue, tandis que la fille parlera de son yaro («son jeune»). Ces relations entre garçons et filles ne sont pas supposées être sérieuses et, du moins dans le passé, elles n'avaient pas vocation à aboutir au mariage. Tout en étant personnelles, librement choisies, elles s'expriment dans un cadre collectif, en présence et souvent par l'intermédiaire des pairs, sans réelle place à l'intimité.

6. Yaro $=$ jeune homme, $m u$ désigne un état, yaromu $=$ état ou manifestation de la jeunesse.

7. Les hommes revendiquent leur capacité à abattre sans fatigue des travaux durs comme la marque de leur entrée dans la jeunesse, tandis que les femmes font référence à la fois aux transformations corporelles et à la capacité à mener de nouvelles activités (cuisine, déplacements en brousse). 
Comme bien d'autres populations africaines ${ }^{8}$, la société boo a toujours ménagé à ses jeunes un temps de liberté sentimentale avant le mariage, tout en étant peu permissive à l'égard de la sexualité et, surtout, de la procréation préconjugale. Les naissances préconjugales avaient un caractère exceptionnel jusqu'à la fin des années 1980; la sexualité préconjugale était sans doute rare, avec, le cas échéant, l'avortement ou le mariage rapide comme réponse à une grossesse préconjugale.

L'espace de liberté juvénile contraste avec les rapports distanciés qui sont de règle au sein du couple marié. L'évitement est d'ailleurs de mise entre jeunes se sachant liés par une promesse de mariage. «[Quand on s'amusait sur la place publique], quand on disait que untel était ton propre fiancé, il n'y avait pas d'amusement entre vous, on se saluait et après tu t'en allais» (Juliette, née en 1965). L'adolescence est une période de jeu, de «vacances» avant le mariage et la prise de responsabilités du statut d'adulte. «En ce moment [de la jeunesse], on ne savait même pas ce que "souci" voulait dire» (Francine, née en 1947). «Jeunesse vaut mieux que manger du mil concassé» dit le proverbe (Leguy, 2001) : c'est un temps béni, sans comparaison avec la vie ordinaire.

Cette organisation très libre de la jeunesse fait cependant partie intégrante de l'encadrement social des jeunes générations par leurs aînées. En effet, pendant que les jeunes s'amusent entre amis (avec la bénédiction des aînés), ils n’ont aucune raison de se préoccuper d'autres affaires, notamment de leur mariage. Il s'agit là d'une question autrement plus sérieuse: «Le mariage n'est pas comme une chasse organisée par des gosses, où n'importe qui peut aller» (proverbe, Leguy, 2001).

\section{Les procédures matrimoniales : une organisation compliquée, un succès incertain}

Ce qui rend le mariage si compliqué est d'abord la formalisation qui l'entoure. Il n'y a pas de prescriptions contraignantes sur le choix du conjoint dans les villages étudiés, plutôt des pratiques d'alliance entre lignages, facilitant la prospection matrimoniale sans être exclusives: l'exigence première est de trouver une épouse à chacun des hommes du lignage.

Classiquement, la procédure pour accéder à une jeune femme célibataire s'étend sur plusieurs années. Elle commence avec l'accord de la famille de la fille, sollicité par le lignage du jeune homme et transmis par un médiateur. La famille de la fille reçoit dès lors des prestations annuelles (travaux agricoles, remise de grains et, aujourd'hui, remise d'argent). Le temps des fiançailles s'achève par un rapt symbolique («enlèvement»), à la suite duquel la fille est confiée («déposée») à une famille alliée ou amie de celle de l'époux, en attendant la fête du mariage. Cette fête sanctionne l'union et honore la nouvelle épouse au sein du village de son époux. Elle marque aussi le début de la cohabitation conjugale.

La procédure mobilise ainsi différents éléments. Elle ne correspond pas pour autant à un agencement figé. Certaines composantes peuvent être renforcées, d'autres retirées

8. Voir par exemple Tabet, 1985; Mair, 1974; Lallemand, 1977; Rondeau, 1994. 
sans que cela joue sur la légitimité de l'union. Par exemple, les prestations ${ }^{9}$ ne conditionnent pas les droits d'un homme sur son épouse et sa descendance ${ }^{10}$. N'ayant pas (ou peu) de valeur d'échange, elles ne sont pas un support nécessaire à la circulation des femmes et ne se prêtent pas non plus à la thésaurisation au profit d'intérêts individuels. L'enjeu est sans doute à chercher ailleurs. Le dispositif matrimonial permet d'inscrire la construction de l'union dans la durée, et d'offrir ainsi une scène à l'expression des échanges entre alliés, à l'exercice de la sociabilité villageoise et de la solidarité lignagère. En manifestant le savoir-faire des responsables familiaux, il légitime aussi leur autorité et rappelle la place de chacun. L'une de nos interlocutrices s'étonne ainsi que l'on évoque l'intervention familiale dans le mariage de son fils: "Comment un jeune seul pourrait-il courtiser une fille et arriver à l'avoir comme épouse? Si c'est au village, toujours il y a la participation des parents» (Manahan, née en 1956).

Cet agencement permet aussi la réinsertion de couples ayant contourné les étapes initiales de la procédure régulière. La mise en œuvre d'un «enlèvement» concerté entre un jeune homme et son élue, avant tout accord préalable de sa famille, est une tactique reconnue lorsque les chances d'obtenir un consentement par le protocole sont faibles: elle oblige les parents de la jeune femme à entendre cette demande en mariage, très généralement relayée sous forme de «demande de pardon» par des médiateurs que mandate la famille du jeune homme. La jeune femme, jusqu’alors hébergée dans un village tenu secret, sera généralement raccompagnée auprès de sa famille, ou la rejoindra par elle-même si ses parents refusent la sollicitation. La démarche d'enlèvement pourra être renouvelée pour signifier la conviction du jeune couple et amener la famille à se plier au choix des intéressés. La communauté villageoise verra généralement d'un œil compréhensif le fait que la famille renonce à s'opposer à une union qu'elle ne souhaitait pas, même si la jeune fille avait déjà été promise: l'événement est interprété comme une dissidence d'ordre individuel dont nul n'est à l'abri. En cas de situation trop conflictuelle pour espérer une entente, il reste au couple la possibilité de partir (dans une grande ville, en Côte d'Ivoire) pour éventuellement revenir au village quelques années plus tard quand la naissance de plusieurs enfants aura, de fait, légitimé l'union. De tels cas de figure sont cependant exceptionnels. En général les cas litigieux ne le restent pas longtemps: soit le couple ne perdure pas, soit il est validé par la famille de la fille ${ }^{11}$. Dans ce dernier cas la gestion de l'union sera ramenée à une forme classique: la « déposition» de la fille se poursuivra jusqu'à la fête du mariage comme après un enlèvement rituel et des prestations pourront éventuellement être établies la même année.

En définitive, l'absence de rigidité dans l'organisation de la procédure matrimoniale contribue au contrôle social des unions: d'un côté, il n'y a pas de recette toute faite à

9. Pour un examen critique des grilles d'interprétation des prestations matrimoniales, voir en particulier Comaroff (1980).

10. L'absence de versement peut au contraire signifier un mariage valorisé, bâti sur une relation antérieure forte d'amitié ou d'alliance.

11. Cet impératif de validation familiale transparaît dans une remarque fréquente des femmes enquêtées: «Si ma famille n’avait pas été d'accord, je ne serais pas ici». 
suivre pour accéder à une épouse, de l'autre, cette souplesse permet de rattraper des écarts individuels et ainsi d'éviter une mise en cause ouverte de l'institution. Mais elle explique aussi la fragilité des procédures matrimoniales: des exigences à géométrie variable ouvrent toujours une brèche à la remise en question du protocole, permettant d'éventuels ajustements et révisions. De fait, le «rendement» des procédures matrimoniales s'avère relativement faible: à peine plus de la moitié aboutissent à un mariage, si l'on se base sur les procédures relatives aux hommes enquêtés, engagées avant 1970 et validées par l'accord de la famille de la femme (Hertrich, 1996). La contestation individuelle (de la femme pour l'essentiel) n'est pas seule en cause: elle est citée dans la moitié des cas, les différends entre familles intervenant par ailleurs dans près d'un quart des cas. L'incertitude de la procédure s'ajoute donc à sa complexité comme facteur dissuassif d'une appropriation individuelle et comme justificatif à la spécialisation des compétences en matière matrimoniale.

\section{La preuve par l'efficacité}

Sans doute le projet des hommes à chercher eux-mêmes leur épouse ne se justifieraitil vraiment que s'ils se sentaient lésés par les démarches de leurs aînés. Mais le système s'avère efficace. La plupart des hommes accèdent à une épouse lorsqu'ils sont relativement jeunes, avant d'avoir eu le temps de s'en inquiéter. La majorité des mariages ont lieu avant l'âge de 25 ans, la moitié avant 22 ans, et le célibat définitif est rare (tableau 1). Il est exceptionnel, chez les Bwa, que les chefs de famille profitent de leur titre pour détourner à leur profit de jeunes épouses, en prolongeant la durée de célibat de leurs cadets. Il est quasiment impensable qu'un homme marie une jeune femme pouvant devenir l'épouse de son fils. La polygamie existe et concerne environ un cinquième des hommes mariés, mais il s'agit d'une pratique relativement instable, en grande partie alimentée par les ruptures d'union (Hertrich, 1996, 2008).

Le contrôle du marché matrimonial a pu être interprété comme un moyen à la disposition des aînés pour contrôler la force de travail des jeunes (Meillassoux, 1975) ${ }^{12}$ : reporter le mariage des hommes permet de reporter leur émancipation économique et de signifier leur dépendance. Cette logique n'est pas de mise chez les Bwa: l'accès à l'autonomie économique n'est pas associé à l'entrée en union. Le couple intègre l'exploitation agricole du mari. Devenir responsable économique n'est pas une étape organisée socialement, elle intervient à un âge variable selon les contingences (décès du responsable, unité devenue très nombreuse, conflit...), mais généralement bien après le mariage $^{13}$. L'organisation économique encourage plutôt les familles à marier rapidement leur fils, car l'arrivée d'une épouse augmente la main d'œuvre et ralentit la pratique migratoire masculine. C'est aussi à l'avantage des mères ainsi libérées par leur bru de la préparation des repas. À l'inverse, il n'y a pas d'intérêt majeur à marier rapidement une fille, sinon celui de limiter une éventuelle contestation du mariage: d'une

\footnotetext{
12. Pour une lecture critique, voir par exemple Guyer (1981) et Comaroff (1980).

13. Vers 35 ans en moyenne, soit une douzaine d'années après le mariage (Hertrich, 1996).
} 
part la jeune fille contribue à l'économie domestique, d'autre part la compensation matrimoniale est modeste et n'a pas vocation à circuler pour trouver des épouses aux fils.

De fait, on n'observe pas d'écart considérable entre l'âge au mariage des hommes et celui des femmes (âge médian): il est de l'ordre de 4 ans, alors qu'il dépasse 8 ans en milieu rural au Mali (République du Mali, 2001; Hertrich, 2007, 2008). Le contraste tient principalement à l'entrée en union des hommes, nettement plus tardive à l'échelle nationale (27 ans en valeur médiane au dernier recensement pour le milieu rural, contre 22 ans dans les villages étudiés), l'âge médian au premier mariage des femmes étant sensiblement identique (de l'ordre de 18 ans).

\section{TENDANCES LONGUES, CHANGEMENTS RÉCENTS}

Finalement le contrôle social de la jeunesse et de la formation des couples est subtil: la mainmise des aînés sur la gestion matrimoniale ressemble plus à un service qu'à une confiscation; la valorisation du statut de jeune fait oublier son titre de mineur... L'encadrement du mariage porte ainsi en soi de quoi désactiver la contestation de l'autorité matrimoniale par les jeunes hommes. Il s'agit là d'un premier facteur contribuant à la reproduction des structures matrimoniales. L'absence de rigidité qui caractérise les exigences de la transaction matrimoniale en constitue un autre: quand la souplesse est de mise, il est possible de s'adapter sans en avoir l'air.

De fait, les biographies matrimoniales et les entretiens confirment la flexibilité qui a toujours existé dans l'encadrement des entrées en union, permettant une prise en compte progressive des attentes individuelles sans remise en cause fondamentale du contrôle formel des mariages. Une rupture semble cependant engagée depuis une dizaine d'années, avec le recul de la formalisation des unions et la diversification de la pratique matrimoniale. On a un premier aperçu de cette articulation entre une tendance longue d'aménagement de la pratique matrimoniale et un virage récent d'une autre nature quand on examine l'appariement conjugal et l'âge au premier mariage (tableau 1). Sur le long terme, on constate une généralisation des couples constitués sur une base "égalitaire» (en âge) depuis les générations anciennes jusqu'à celles qui se sont mariées dans les années 1980: la plupart des jeunes (près de $90 \%$ chez les hommes et près de $80 \%$ chez les femmes) débutent désormais leur vie conjugale avec un conjoint également célibataire, d'un âge souvent proche du leur. Le rapprochement du marché matrimonial des deux sexes s'est d'abord traduit par un accès plus rapide aux épouses (baisse de l'âge au mariage des hommes et de l'écart d'âge au mariage entre sexes) avant le retournement du début des années 1990. Le recul de l'entrée en union touche de façon semblable les femmes et les hommes (augmentation de l'âge médian au premier mariage d'environ une année et demi) et coïncide avec l'essor des migrations des jeunes filles. Ces tendances, leur signification et leur articulation peuvent être abordées de manière plus approfondie en cadrant l'analyse sur les unions entre célibataires et les procédures matrimoniales qu'elles ont mobilisées. 
Tableau 1 L'entrée en union. Âge au mariage et appariement conjugal

\begin{tabular}{|c|c|c|c|c|c|c|c|}
\hline \multirow[b]{2}{*}{ Indicateurs } & \multicolumn{7}{|c|}{ Générations } \\
\hline & $\begin{array}{c}\text { avant } \\
1944\end{array}$ & $1944-58$ & $1959-68$ & $1969-73$ & $1974-78$ & $1979-83$ & $1984-88$ \\
\hline \multicolumn{8}{|c|}{ Âge médian au premier mariage } \\
\hline Hommes & 22,7 & 21,7 & 21,3 & 22,4 & 23,5 & 22,6 & - \\
\hline Femmes & 18,0 & 17,6 & 17,7 & 18,5 & 19,4 & 18,7 & $19,2^{* *}$ \\
\hline Écart & 4,7 & 4,1 & 3,6 & 3,9 & 4,1 & 3,9 & - \\
\hline \multicolumn{8}{|c|}{ Proportion (\%) d'individus ayant conclu leur premier mariage avec un(e) célibataire } \\
\hline Hommes & 72 & 77 & 90 & 85 & $89 *$ & - & - \\
\hline Femmes & 64 & 69 & 64 & 78 & 81 & $81 *$ & - \\
\hline \multicolumn{8}{|c|}{ Proportion (\%) de célibataires au dernier passage de l'enquête (2004) } \\
\hline Hommes & 5 & o & 3 & 7 & 24 & 53 & - \\
\hline Femmes & o & ○ & ○ & ○ & 2 & 13 & 75 \\
\hline \multicolumn{8}{|l|}{ Effectifs } \\
\hline Hommes & 98 & 96 & 94 & 82 & 94 & 140 & 179 \\
\hline Femmes & 113 & 113 & 123 & 85 & 116 & 156 & 170 \\
\hline
\end{tabular}

* Estimation.

Source: Enquête biographique, individus enquêtés au titre de résidants à l'un des passages (1987-89, 1995, 2000, 2004).

\section{Dynamiques décisionnelles}

Si la recherche d'épouses relève de la responsabilité des lignages, elle n'exclut pas la participation des jeunes hommes concernés. La prospection matrimoniale se donne plutôt à lire comme une affaire qui mobilise toutes les bonnes volontés; le repérage d'une future épouse pourra par exemple être le fait d'une femme originaire du lignage du jeune homme, mariée dans le village ou le lignage de la jeune fille. Les pères pourront, le cas échéant, suggérer à leurs fils d'aller avec leurs amis entourer et courtiser la jeune fille pour promouvoir le projet matrimonial. Réciproquement, ils pourront prendre acte des affinités qui naissent dans le cadre des activités de jeunesse et chercher à les relayer dans un cadre formel. Ainsi la frontière théorique entre le champ matrimonial contrôlé par les aînés et l'espace ludique des jeunes présente une certaine porosité, à laquelle le pragmatisme des acteurs familiaux n'est pas étranger. On en trouve l'expression dans les entretiens qualitatifs, y compris auprès des hommes les plus âgés (nés dans les années 1930). De fait, l'implication du jeune homme dans la préparation de son union existe de longue date, mais sans empiéter sur la gestion formelle de l'union réservée aux aînés. Cette implication s'est accrue au fil des générations, dans le cadre d'une concertation familiale croissante: de l'initiative lignagère annoncée à l'intéressé au choix personnel que le jeune homme soumet à sa famille et lui demande de relayer formellement, les échanges autour du projet matrimonial prennent des formes et des issues variées. L'encadré 1 en donne un aperçu au travers des initiatives qui ont présidé aux mariages de Jean-Noël et de ses 6 premiers enfants: on observe une présence 
toujours plus affirmée et plus précoce du jeune dans le choix de l'épouse, mais qui n'écarte jamais complètement la contribution parentale. Au sein des générations les plus âgées de notre enquête, l'initiative individuelle ${ }^{14}$ concernait déjà près d'1 mariage sur 3. Elle intervenait dans environ la moitié des mariages entre célibataires des années 1970-1990, et en concerne aujourd'hui la majorité (80 \%) (tableau 2). Les attentes personnelles masculines se sont principalement exprimées dans les coulisses familiales, sans contestation apparente du rôle des responsables familiaux dans la gestion matrimoniale. Cela du moins jusqu'à la fin des années 1980: dans la plupart des cas (7 à 8 fois sur 10), les mariages d'initiative individuelle faisaient l'objet d'une procédure classique gérée par les aînés, contre la moitié au cours des années récentes (tableau 2). L'intervention des intéressés s'articule ainsi à l'ouverture d'un espace de concertation et d'échange interindividuel au sein de la famille, à une certaine privatisation des rapports familiaux et du projet matrimonial. On en trouve une autre expression dans la raréfaction des initiatives matrimoniales relevant de la parenté élargie, deux fois plus rares chez les jeunes générations que chez les anciennes (tableau 2), tandis que l'intervention des parents proches (père, mère, frère) est restée stable jusqu'à une date récente. Le lieu d'initiative de l'union s'est à la fois ouvert à l'intéressé et resserré sur son environnement familial proche.

Tableau 2 Décision de l'union: initiative et mode d'accès à l'épouse

\begin{tabular}{|c|c|c|c|c|c|}
\hline \multirow[b]{2}{*}{ Indicateurs } & \multicolumn{5}{|c|}{ Générations } \\
\hline & $\begin{array}{c}\text { avant } \\
1944\end{array}$ & $1944-58$ & $1959-68$ & $1969-73$ & $1974-78$ \\
\hline \multicolumn{6}{|c|}{ Initiative de l'union (relation de parenté entre l'initiateur et l'époux) } \\
\hline Ego & 33 & 49 & 54 & 49 & 79 \\
\hline Parents proches (père, mère, frère) & 37 & 29 & 31 & 36 & 8 \\
\hline \multirow[t]{2}{*}{ Autre } & 30 & 22 & 15 & 15 & 13 \\
\hline & 100 & 100 & 100 & 100 & 100 \\
\hline \multicolumn{6}{|l|}{ Mode d'accès à l'épouse } \\
\hline Accord de la famille de la fille & 84 & 86 & 70 & 66 & 51 \\
\hline \multirow[t]{2}{*}{ Sans accord préalable à «l'enlèvement » } & 16 & 14 & 30 & 34 & 49 \\
\hline & 100 & 100 & 100 & 100 & 100 \\
\hline \multicolumn{6}{|l|}{ Proportion (\%) de mariages d'initiative } \\
\hline $\begin{array}{l}\text { individuelle ayant donné lieu à une procédure } \\
\text { avec accord initial de la famille de la fille }\end{array}$ & 70 & 80 & 66 & 56 & 48 \\
\hline Nombre d'observations & 32 & 42 & 56 & 56 & 55 \\
\hline
\end{tabular}

Source: Enquête biographique, hommes enquêtés au titre de résidants à l'un des passages (1987-89, 1995, 2000, 2004).

Champ: Premiers mariages des hommes avec une femme célibataire.

14. La question a été posée sous la forme suivante: «Au départ qui a eu l'idée de cette femme pour toi?». La réponse nominative a ensuite été saisie en identifiant la relation de parenté entre la personne citée et l'enquêté. 
Si la participation du jeune homme au choix de son épouse a pu croître progressivement sans poser problème, cela tient sans doute à la fois au caractère parfaitement conciliable des niveaux d'intervention (choix personnel, visibilité collective) et à la primauté d'un objectif partagé (trouver une femme). Il n'en est pas de même pour la participation féminine à la décision matrimoniale. La construction de l'échange matrimonial ne place pas l'initiative du côté féminin (on cherche des femmes, on ne cherche pas des hommes); l'intervention de la famille de la femme à la décision matrimoniale est plus passive, correspondant à l'acceptation ou au refus d'une sollicitation. Un refus, même d'origine familiale, peut être difficile à exprimer surtout si les lignages sont déjà liés par des échanges matrimoniaux; une motivation personnelle a d'autant moins de chance d'être reconnue...

La famille est supposée consulter l'intéressée avant de répondre favorablement à une demande matrimoniale (Capron 1973, 1988b). Cependant il semble bien que cette étape ait souvent été contournée ou réduite à une dimension symbolique. Dans les entretiens qualitatifs, un tiers des femmes des différentes générations disent ne pas avoir été consultées avant d'avoir été promises ${ }^{15}$. Certaines n'ont appris que tardivement l'engagement pris par leurs parents. Ainsi Soiniwé (née en 1931, mariée en 1953) a-t-elle été surprise quand son père lui a dit qu'elle épouserait Zunfo: «Je ne m’y attendais pas car j'ai toujours cru que les arachides qu'on amenait chez nous étaient pour le mariage de ma grande sœur. [...] Intérieurement je ne voulais pas de ce mariage. Mais je devais obéir à mes parents. En ce temps-là les jeunes filles étaient frappées si elles osaient s'opposer.» Même quand elle est consultée, la jeune femme a souvent du mal à exprimer ses réticences, plus encore à les faire entendre. Rosalie (née en 1940) se rappelle avoir vainement essayé de convaincre ses parents d'accepter son yaro: «... comme c'est mon père qui organisait le mariage, je ne pouvais pas désobéir [...] je n’avais plus de choix à faire, sauf le choix de mes parents.» Aujourd'hui, de telles situations sont devenues plus rares ${ }^{16}$, sans avoir complètement disparu pour autant. Ainsi en est-il de Yisouara (née en 1981, mariée en 1998): «Je ne savais même pas pour qui on m’enlevait. C'est plus tard que j'ai compris que j'étais là pour Mimanu. Je ne l'aimais pas mais je ne pouvais pas m’enfuir, je ne connaissais pas le village où on m’avait déposée. En plus mes parents avaient donné leur accord sans me demander mon avis. Je ne pouvais que rester.» La mémoire collective rappelle cependant volontiers l'histoire de couples qui se sont constitués sur des bases amoureuses, en dépit de l'opposition familiale, même parmi les générations anciennes. Comme on l'a signalé, le fait de fuir vers l'étranger ou plus modestement d'organiser un enlèvement symbolique en cachant la jeune fille

15. L'implication tardive des femmes au projet matrimonial, en particulier pour les anciennes générations, ressort également d'une comparaison des déclarations masculines et féminines sur le détail des processus matrimoniaux: les femmes décrivent des procédures plus courtes et moins complexes que leur conjoint; elles en ont une connaissance "tronquée», se rapportant plus souvent aux dernières étapes de la démarche matrimoniale (Hertrich, 1997).

16. Parmi les femmes enquêtées ayant conclu leur premier mariage au cours des années 1995-2004, plus de $90 \%$ déclarent avoir été partie prenante de l'initiative de l'union ou avoir été contentes à la perspective de leur mariage. 
dans un village voisin pouvait permettre au jeune couple de passer outre un refus officiel, en attendant qu'une «demande de pardon» trouve finalement satisfaction ou, à défaut, que l'éloignement et la naissance d'un enfant apaisent les esprits. Les mariages conclus sans avoir fait l'objet d'un accord préalable à l'enlèvement fournissent, au sein des anciennes générations, une approximation ${ }^{17}$ des unions décidées par les individus en forçant l'autorité familiale. 1 mariage sur 7 correspondait à ce cas de figure avant les années 1980 (hommes nés avant 1959). Cet indicateur atteint un niveau deux fois plus élevé pour les mariages des années 1980-1995 et passe à 50 \% pour les unions des dernières années. On observe donc au sein des deux partis de l'alliance un même recul de l'implication familiale dans la décision de l'union, qui s'est encore récemment accéléré. La multiplication des unions non initiées par l'accord de la famille de la fille peut être signe d'une émancipation féminine de la tutelle matrimoniale. Mais de façon moins directe qu'on ne pourrait le penser, car en se banalisant ce mode d'accès à l'épouse a aussi changé de signification. Autrefois il traduisait une contestation et une mise en échec de l'autorité familiale. Aujourd'hui ${ }^{18}$ il traduit davantage une moindre exigence de contrôle matrimonial des familles face à une jeunesse féminine de moins en moins présente au village. Plus qu'une revendication féminine explicite portant sur le choix du conjoint, il semble que ce soit la migration des filles en ville, comme on le verra plus loin, qui a ouvert une brèche dans l'organisation de la gestion matrimoniale, en amenant les responsables familiaux à douter de leur aptitude à observer leurs engagements, avant même le refus clair des intéressées. Pour autant, même tardive et d'ordre symbolique, la reconnaissance familiale reste importante dans la validation d'une union, et il est difficile de s'en émanciper, ne serait-ce que psychologiquement. Reine (encadré 1) l'a appris à ses dépens, trop mal(ade) pour vivre sereinement sa vie de couple avant d'avoir reçu la bénédiction paternelle.

Encadré 1 Les mariages de Jean-Noël et de ses enfants

Né en 1947, cadet et seul garçon de sa fratrie (6 enfants survivants), Jean-Noël a eu une jeunesse facile, choyé par ses parents et ses sœurs. Il s'est marié jeune, très jeune ( «je doutais de moi-même, je me demandais si j'avais vraiment l'âge de me marier») avec Francine, du même âge que lui, 17 ans. C'est sa sœur Paru, de 3 ans son aînée, qui en a eu l'idée; épouse d'un homme du lignage de Francine, elle avait vu combien cette dernière était travailleuse et pouvait constituer un bon parti. JeanNoël, qui avait commencé à vivre sa jeunesse et à courtiser, a suivi les conseils de sa sœur et a reporté son attention sur Francine. «Elle m’a dit “si tu pouvais avoir cette

17. Approximation probablement surestimée car certaines unions concertées de façon informelle entre familles peuvent également prendre cette tournure. Voir par exemple le cas d'André, donné dans l'encadré 1 .

18. L'absence d'accord avant le rapt symbolique n'est plus synonyme de tension relationnelle avec la famille de la fille, pas plus qu'elle ne signifie forcément l'engagement de la jeune femme dans le choix de son conjoint (le cas de Yisouara, cité plus haut, en témoigne). 
fille cela me ferait plaisir" [...]. J'avais d'autres filles en vue mais ma sœur m'a dit que cela ne lui plaisait pas [...] et comme il n'y avait rien de sérieux entre moi et ces filles [...], j'ai vu qu'elle [Francine] m'intéressait plus que les autres.» Les causeries du soir entre jeunes étaient l'occasion de manifester ses sentiments, mais de façon indirecte, en passant par les amis. «D’un côté c'était de l'amusement parce qu'on se racontait des histoires, des devinettes [...]. Le sérieux venait plutôt après les histoires, par exemple mon ami pouvait demander à ma hazunu: "Maintenant parlons. Est-ce que tu aimes mon ami?" Et, si elle disait oui: “Mais est-ce que tu n'as pas l'âge du mariage? Dans combien de temps est-ce qu'on peut t'enlever?”... Tout ça.» Parallèlement, les parents de Jean-Noël ont lancé la procédure en recourant aux services d'un griot pour la médiation interlignagère; l'accord de la famille de Francine a ainsi été recueilli. Deux années de suite, un groupe de travail d'une quinzaine d'hommes a été mobilisé pour faire la récolte du mil dans les champs de la famille de Francine, et on a donné de l'arachide. Une première tentative «d'enlèvement» a alors eu lieu mais c'était trop tôt, Francine a fui; la deuxième tentative un an plus tard a été la bonne. La jeune femme a d'abord été confiée à la mission catholique, à une trentaine de kilomètres du village, puis encore 3 semaines au village. Le mariage a alors été fêté et le couple s'est installé dans une case inhabitée du village avant de construire la sienne. Pour la fête, «il n'y avait pas beaucoup de dépenses en ce tempslà. Il y a eu 2 cabarets de bière de mil, 4 chèvres et on a préparé le tô [plat de mil], plus l'argent pour les griots.» Les moyens ont été fournis par la famille: la «grande famille» (lignage) s'est cotisée pour fournir le mil du tô et de l'un des cabarets, le père et la mère de Jean-Noël ont fourni les chèvres, la boisson du deuxième cabaret a été fabriquée à partir du mil de l'exploitation agricole. À cela s'ajoutent les contributions (en argent ou en nature) des villageois et des membres de la famille destinées à honorer la jeune épouse et à l'équiper en ustensiles de cuisine et en vêtements. Catholiques tous les deux, Jean-Noël et Francine ont également fait la même année un mariage «chez les Pères» et, par la même occasion, un mariage civil.

Le couple a eu 13 enfants, dont 8 sont en vie et 6 sont mariés. Jean-Noël n'a pas pris d'initiative de son propre chef pour procurer des épouses à ses fils: «Cela n'est plus forcé de nos jours. C'est quand ton fils te dit qu'il veut telle fille que tu t'y mets, que tu l'aides pour le reste.» Les unions de ses enfants concilient ainsi à des degrés variables l'intervention familiale et la démarche personnelle.

L'aîné, Denis (né en 1965), avait commencé à courtiser une jeune femme quand il a décidé de partir travailler en Côte d'Ivoire. Il a alors confié l'affaire à son père. «Il m'a dit “je connais une fille, fais tout pour que je puisse l'avoir". Quand il est parti, je suis allé parler aux parents, ils ont accepté, j'ai fait le nécessaire jusqu'à ce qu'on l'ait eue.» Il y a eu 3 années de travaux agricoles, la remise d'arachides et d'une somme de 13000 francs CFA. Selon la formule consacrée, la jeune femme a été enlevée puis déposée avant de rejoindre son époux en Côte d'Ivoire en 1986, accompagnée de son beau-père. L'union n'a donné lieu à aucune cérémonie. 
André (né en 1970) aurait pu se marier selon la même procédure que son grand frère, en espérant que son père lui obtienne la jeune fille de son choix. «Il m'a montré une fille et j'ai essayé de faire les démarches mais cela n’a pas marché. Par la suite, il a eu une fille par lui-même.» Après 3 ans de séjour en Côte d'Ivoire, il revient au pays en 1992 et s'entend avec Hiéhan, une jeune femme d'un village proche récemment revenue de Ségou. Il l'enlève et la dépose dans un village voisin tandis qu'un griot est envoyé auprès de la famille de Hiéhan pour «demander le pardon». La famille accepte la demande malgré l'accord donné quelques mois plus tôt à un autre candidat, accompagné d'un début de prestation (5 000 francs CFA). Cette union ne donnera lieu à aucune prestation mais elle sera fêtée au village et fera l'objet d'un mariage religieux et civil la même année.

Robert (né en 1972) a eu son épouse en 1993 en saisissant une opportunité offerte par son père. "Quelqu'un est venu m'offrir sa fille, il a dit “j'ai une fille je te la donne”. Moi j'ai dit ça à mes fils "si l'un d'entre vous aime cette fille...”. Robert seul avait l'âge de se marier, il a dit qu'il était d'accord donc c'est à lui qu'on l'a donnée.» Robert et ses amis ont ainsi organisé l'enlèvement de Mwahan et l'ont déposée directement au village dans leur lignage. Le pardon a été demandé et une partie de travail a été réalisée lors de la saison agricole suivante. Le mariage a été fêté au village et il y a eu un mariage civil l'année suivante, mais pas de mariage religieux, Mwahan n'étant pas chrétienne. On a ici le cas d'un mariage concerté entre les pères des jeunes gens mais qui a toutes les apparences d'une démarche en marge des cadres réguliers: c'est que la concertation des pères a été directe, contournant la formalisation de l'échange interlignager.

Julien (né en 1974) et Jacques (né en 1978) ne se sont quant à eux adressés à leur père qu'après avoir «enlevé» leur élue, afin d'effectuer la demande de pardon. Pour Jérôme, «c'était une fille [du village] qui était revenue de la ville, Jérôme l'a courtisée sans que je sois au courant [...], c'est quand ils l'ont enlevée qu'il est venu me le dire». Fête au village, mariage légal et mariage religieux ont eu lieu dans la foulée, en 1997. Quelques prestations (de l'arachide et 11000 francs CFA) ont été versées après le mariage. L'épouse de Jacques, Manahan, est fille de voisins et Jean-Noël pensait que le mariage se ferait facilement. Les deux jeunes s'appréciaient et avaient eu le temps de se connaître au village et à Bamako, lorsqu'ils y étaient en migration de travail. L'affaire a été plus compliquée que prévu car, sans s'opposer ouvertement, les parents n'ont cessé de reporter l'union de leur fille. «Au commencement les parents de la fille pensaient que c'était seulement de l'amusement et que cela n'allait pas aboutir. Quand c'est devenu sérieux, ils n'ont pas voulu dire carrément qu'ils ne voulaient pas, c'est pourquoi ils ont créé cette situation pour que cela se gâte. » Après 3 enlèvements et une remise de 25000 francs CFA, les parents de Manahan finiront par se résigner, mais la fête du mariage, de même que les mariages légal et catholique, ne seront organisés qu'un an plus tard, en 2000, quand les différends familiaux se seront réglés. 
Jean-Noël a également une fille, Reine (née en 1976), qui s'est mariée en ville en 1998 sans associer de quelque façon ses parents, une négligence qu’il lui a été nécessaire de corriger pour vivre sereinement. «Quand elle est partie, je lui avais bien dit qu'il ne fallait pas qu'elle se marie là-bas. Elle a quand même connu l'homme là-bas et elle s'est cachée pour déménager chez le monsieur. Mais entre-temps elle est tombée malade et elle s'est dit que peut-être que c'était parce qu'elle n'avait pas respecté les paroles de ses parents. Elle est revenue vite et elle a dit qu'elle avait trouvé un mari. [...] Ensuite elle a guéri.»

\section{Des procédures de plus en plus courtes, de moins en moins contraignantes}

L'évolution à long terme de l'organisation matrimoniale, par ajustement permanent sans remise en question brusque, ressort également de l'analyse des prestations matrimoniales. La proportion de mariages ayant donné lieu à des prestations est restée relativement stable au fil du temps, autour de $80 \%$ (tableau 3). Un certain équilibre semble également s'être établi entre les contributions en nature (travail et grain) et les contributions monétaires apparues dans les années 1960-1970 (remise d'argent ou achat au prix fort de bière de mil confectionnée par la famille de la fille), les unes et les autres intervenant dans une proportion sensiblement identique des mariages, autour de $60 \%$.

On pourrait donc croire que les responsables familiaux ont gardé une mainmise sur le marché matrimonial par le contrôle des ressources échangées, voire que la souplesse accordée pour le choix du conjoint est contrebalancée par des exigences plus élevées en termes de redevances matrimoniales. Une inflation des prestations a ainsi été constatée dans de nombreuses sociétés subsahariennes ${ }^{19}$; la difficulté à réunir la somme de la compensation est également citée comme facteur d'augmentation de l'âge au mariage, notamment en milieu urbain ${ }^{20}$. Une telle grille de lecture perd cependant toute validité quand on examine le détail des prestations versées (tableau 3).

Certes les prestations agricoles se sont maintenues mais elles sont désormais réduites a minima. Le service agricole répété plusieurs années consécutives a quasiment disparu: il concernait la moitié des hommes nés avant 1944, 1 sur 5 parmi les générations nées dans les années 1950 et 1960 et à peine 1 sur 10 pour les générations plus récentes. La remise d'arachides ou de céréales est également devenue moins fréquente, concernant moins de la moitié des unions à partir des générations nées dans les années 1960 et un cinquième pour celles nées à la fin des années 1970.

L'argent est devenu tout aussi fréquent que les prestations en nature et contribue à la perception par les villageois d'un coût aujourd'hui plus élevé pour accéder aux femmes. Cependant, la proportion de mariages concernés n’a pas augmenté au cours des 30 dernières années, le recours à un mode détourné de prestation monétaire (par

19. Voir par exemple Enel et al., 1994; Guigou, 1992; Isiugo-Abanihe, 1994, 1995; Locoh, 1994; Nagashima, 1987.

20. Voir par exemple Antoine et al., 2001; Calvès, 2007; Marcoux et Piché, 1998. 
la vente de bière de mil) initié dans les années 1970 a quasiment disparu et, surtout, les montants remis restent modestes. Même pour les générations les plus jeunes, ils sont inférieurs à 15000 francs CFA dans 70 \% des cas. Une telle somme n’est pas négligeable dans le contexte étudié, mais elle est accessible à un cultivateur moyen et sans commune mesure avec les compensations matrimoniales enregistrées dans d'autres populations de la région, qui peuvent se compter en centaines de milliers de francs CFA (Guigou, 1992; Enel et al., 1994). On ne peut donc pas considérer les prestations monétaires comme la marque d'une réaffirmation de l'autorité familiale dans le champ matrimonial. On en a une autre preuve en en examinant l'affectation: celles-ci sont le plus souvent destinées à la jeune femme ( 3 fois sur 4 d'après les déclarations des femmes enquêtées).

Tableau 3 Prestations matrimoniales

\begin{tabular}{|c|c|c|c|c|c|}
\hline \multirow[b]{2}{*}{ Indicateurs } & \multicolumn{5}{|c|}{ Générations } \\
\hline & $\begin{array}{c}\text { avant } \\
1944\end{array}$ & $1944-58$ & $1959-68$ & $1969-73$ & $1974-78$ \\
\hline Existence de prestations (\% de mariages) & 63 & 88 & 79 & 75 & 78 \\
\hline \multicolumn{6}{|l|}{ Prestations en nature } \\
\hline \multicolumn{6}{|c|}{ Répartition (\%) des mariages selon le nombre d'années de travaux agricoles } \\
\hline o & 44 & 36 & 39 & 53 & 63 \\
\hline 1 & 6 & 43 & 39 & 35 & 26 \\
\hline 2 & 22 & 7 & 14 & 13 & 11 \\
\hline \multirow[t]{2}{*}{3 et plus } & 28 & 14 & 7 & o & o \\
\hline & 100 & 100 & 100 & 100 & 100 \\
\hline Proportion (\%) de mariages avec remise de grains & 53 & 64 & 41 & 41 & 20 \\
\hline $\begin{array}{l}\text { Proportion (\%) de mariages avec remise de grains } \\
\text { ou travaux agricoles }\end{array}$ & 63 & 79 & 63 & 58 & 50 \\
\hline \multicolumn{6}{|l|}{ Prestations monétaires } \\
\hline $\begin{array}{l}\text { Proportion (\%) de mariages avec remise directe } \\
\text { d'argent }\end{array}$ & 13 & 51 & 51 & 56 & 60 \\
\hline $\begin{array}{l}\text { Proportion (\%) de mariages avec achat de bière } \\
\text { de mil }\end{array}$ & 14 & 25 & 21 & 7 & 4 \\
\hline $\begin{array}{l}\text { Proportion (\%) de mariages avec remise d'argent } \\
\text { ou achat de bière de mil }\end{array}$ & 21 & 61 & 62 & 56 & 60 \\
\hline
\end{tabular}

Source: Enquête biographique, hommes enquêtés au titre de résidants à l'un des passages (1987-89, 1995, 2000, 2004).

Champ: Premiers mariages des hommes conclus avec une femme célibataire.

L'évolution observée ne correspond donc pas à un abandon de la redevance matrimoniale, mais à un recentrage sur des formes de prestations qui peuvent être concentrées dans le temps, éventuellement versées en une seule fois. En quelque sorte, la remise de prestations ne constitue plus forcément une longue procédure impliquant une demande d'accord et des échanges préalables à l'union: les prestations peuvent aujour- 
d'hui être concomitantes de l'accès à l'épouse, la demande de pardon s'accompagnant alors d'une remise d'argent ou se traduisant par un service agricole au cours de la saison suivante ${ }^{21}$. De fait, la durée de la procédure matrimoniale a été considérablement écourtée (figure 1). Les processus de 3 ans et plus (durée écoulée entre la demande d'accord et l'accès à l'épouse) autrefois majoritaires sont devenus exceptionnels (moins de $10 \%$ des mariages contre près de $60 \%$ ). Deux tiers des hommes des jeunes générations (contre un quart des plus anciennes) se sont mariés sans avoir engagé de procédure (accord préalable à l'enlèvement) ou en ayant engagé une procédure très courte (de moins d'un an).

Si le maintien de prestations permet de conserver un support d'interaction interlignagère à l'occasion du mariage, cette dernière se trouve désormais extrêmement réduite. L'évolution des prestations dessert ainsi doublement les responsables familiaux: ils en tirent moins de bénéfices puisque celles dont ils pouvaient directement profiter (les travaux agricoles) se sont raréfiées; surtout, ils ont perdu l'avantage que leur procurait la longue durée des anciennes procédures, qui montrait et légitimait leur compétence et leur autorité.

\section{Figure 1 Durée du processus matrimonial, selon la génération de l'homme}

Durée écoulée entre la demande d'accord et la cohabitation

Premiers mariages des hommes conclus avec une femme célibataire

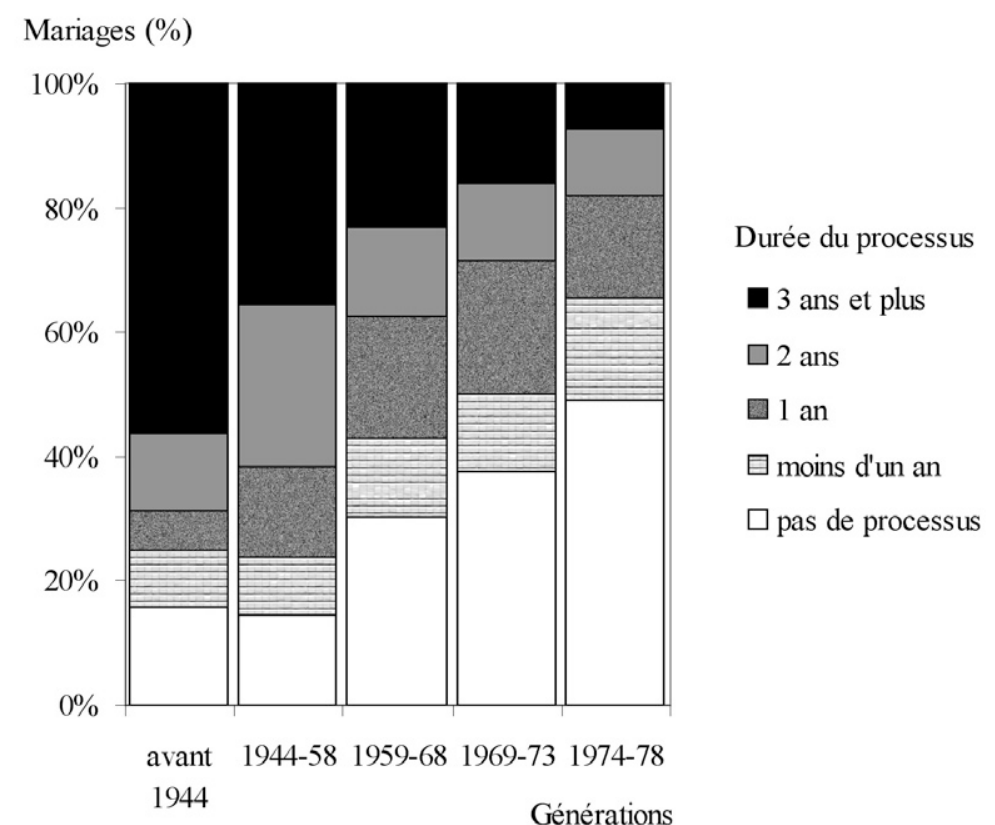

21. Sur la décennie 1994-2003, on enregistre un versement de prestations après la cohabitation pour plus de la moitié des mariages avec prestations. On ne dispose pas d'estimation quantitative sur les périodes antérieures (la question ayant été introduite dans l'enquête à partir du passage de 2000), mais tout laisse à penser que ces cas étaient rares. 


\section{Une visibilité collective remise en question}

Parallèlement à la perte de visibilité subie par les responsables familiaux avec la concentration des interventions matrimoniales autour du moment du mariage, on constate des changements dans la participation de la communauté villageoise à la mise en œuvre de l'union. Le tableau 4 permet d'en juger au travers de 3 champs d'intervention classiques d'instances communautaires: le médiateur envoyé auprès de la famille de la fille lors de la première demande d'accord (ou, à défaut, de pardon), la pratique de la déposition et la célébration du mariage.

Tableau 4 Médiation et sanction sociale de l'union

\begin{tabular}{|c|c|c|c|c|c|}
\hline \multirow[b]{2}{*}{ Indicateurs } & \multicolumn{5}{|c|}{ Générations } \\
\hline & $\begin{array}{c}\text { avant } \\
1944\end{array}$ & $1944-58$ & $1959-68$ & $1969-73$ & $1974-78$ \\
\hline \multicolumn{6}{|l|}{ Médiation (lors de la demande d'accord ou de pardon) } \\
\hline $\begin{array}{l}\text { Proportion (\%) de mariages médiatisés par } \\
\text { un homme de caste }\end{array}$ & 58 & 55 & 62 & 70 & 75 \\
\hline $\begin{array}{l}\text { Proportion (\%) de mariages médiatisés par } \\
\text { un membre du patrilignage de l'homme }\end{array}$ & 26 & 28 & 12 & 7 & 6 \\
\hline \multicolumn{6}{|l|}{ Déposition } \\
\hline Proportion (\%) de mariages avec déposition & 94 & 90 & 80 & 64 & 73 \\
\hline $\begin{array}{l}\text { Dépositions d'une durée inférieure à } 3 \text { semaines } \\
\text { (\% parmi les mariages avec déposition) }\end{array}$ & 18 & 14 & 11 & 29 & 44 \\
\hline \multicolumn{6}{|l|}{ Cérémonies } \\
\hline $\begin{array}{l}\text { Proportion (\%) de mariages ayant donné lieu } \\
\text { à une fête }\end{array}$ & 94 & 93 & 70 & 49 & 40 \\
\hline $\begin{array}{l}\text { Proportion (\%) de mariages ayant donné lieu } \\
\text { à un mariage légal }\end{array}$ & 26 & 31 & 20 & 23 & 15 \\
\hline Effectifs & 32 & 42 & 56 & 56 & 55 \\
\hline
\end{tabular}

Source: Enquête biographique, hommes enquêtés au titre de résidants à l'un des passages (1987-89, 1995, 2000, 2004).

Champ: Premiers mariages des hommes conclus avec une femme célibataire.

Le recours à des hommes de caste pour médiatiser les échanges entre les deux familles reste de mise et pourrait même avoir augmentée ${ }^{22}$ si l'on en croit les indicateurs statistiques (tableau 4). Ainsi, ce n'est pas parce que l'échange d'accord entre familles est de plus en plus souvent reporté après le rituel de l'enlèvement (tableau 2) que cette conciliation se fait de façon directe entre les familles des intéressés. Au contraire, le recours à un intermédiaire appartenant au lignage de l'homme est devenu

22. L'hypothèse d'une augmentation doit cependant être envisagée avec prudence. Les familles peuvent recourir à différents médiateurs au cours d'une même procédure, or l'enquête n'en enregistre qu'un (le premier médiateur sollicité). Si les hommes de caste sont davantage sollicités à mesure que l'on s'approche du mariage (ce qui est vraisemblable), on observera logiquement qu'ils sont d'autant plus souvent cités que le processus est court, ce qui est le cas des jeunes générations par rapport aux plus anciennes. Cela ne signifie donc pas nécessairement une augmentation du recours aux médiateurs de caste. 
exceptionnel, concernant moins d' 1 mariage sur 10 aujourd'hui contre plus d' 1 sur 4 chez les anciennes générations. Paradoxale au premier abord, cette évolution exprime sans doute une privatisation de l'affaire matrimoniale au sein des lignages, tout comme la raréfaction des unions initiées par la parenté élargie (tableau 2). Le réseau lignager est moins mobilisé, peut-être parce que son implication dans la décision matrimoniale diminue, peut-être parce qu'on le sollicite moins, peut-être aussi parce qu'on craint plus les conflits d'intérêts internes, déjà visibles sur le plan foncier. Les hommes de caste sont à cet égard les intermédiaires les plus neutres, donc les plus sûrs: du fait de l'endogamie de caste, toujours respectée, leurs intérêts ne peuvent interférer avec ceux de leurs commanditaires. C'est peut-être davantage leur position «hors système» que leur fonction de délégué de la communauté qui explique le maintien, voire le renforcement du rôle de ces hommes de caste dans la gestion matrimoniale.

En effet, l'implication de la communauté villageoise dans la conclusion des unions et, plus largement, dans la "présentation» ou mise en scène du mariage dans l'espace villageois diminue considérablement depuis deux décennies, comme en atteste l'évolution de la pratique relative à la déposition et, plus encore, celle de la fête du mariage au village. Jusqu'au début des années 1980, ces deux étapes étaient quasi systématiques, même en cas d'absence d'accord familial initial ${ }^{23}$. La pratique de la déposition a commencé à s'éroder il y a une vingtaine d'années; elle est devenue moins systématique et beaucoup plus courte. Les confiages d'une durée inférieure à 3 semaines, rares autrefois ( 1 cas sur 7) couvrent aujourd'hui près de la moitié des cas.

Mais c'est surtout dans la chute spectaculaire du nombre de mariages fêtés au village que l'on constate la baisse de l'encadrement communautaire des unions. À la fin des années 1990, 6 mariages sur 10 ne sont pas fêtés, alors que cette situation était exceptionnelle (7\%) 20 ans plus tôt et encore minoritaire (30 \%) dans les années 1980 (figure 2, tableau 4). Cette baisse n'est pas associée à l'apparition d'autres formes de validation. Le recours au mariage civil a peu évolué au fil du temps et reste minoritaire (environ 1 mariage sur 5) (tableau 4).

En revanche, il semble bien que la fête du mariage, en devenant plus rare, revête aussi de nouvelles formes. Littéralement nommée «fête de la fiancée», sa vocation initiale est d'accueillir et d'honorer la nouvelle épouse en mobilisant la communauté lignagère, mais aussi plus largement la communauté villageoise. Les membres du patrilignage s'investissent dans l'organisation de cette fête à double titre: d'une part en apportant des ressources matérielles et humaines (mise en commun de céréales et d'animaux pour la nourriture et la boisson; organisation d'un service de travail collectif, des femmes pour préparer la bière de mil et le repas de la fête, des jeunes pour chercher

23. On peut rappeler que la «déposition» fait suite au rapt symbolique. C'est une période au cours de laquelle la jeune fille est confiée à une famille alliée ou amie (environ un mois), le temps pour les familles de tenir les derniers conciliabules et d'organiser la «fête de la fiancée» qui rendra public son changement de statut. Pendant ce temps, la jeune fille prend connaissance de son nouvel environnement social et reçoit l'accueil des villageois, en particulier des jeunes qui pour l'honorer viennent l'entourer lors des causeries du soir. 
en brousse le bois nécessaire à la cuisson de ces préparations); d'autre part en constituant un trousseau (pagnes et ustensiles de cuisine) pour la nouvelle épouse. L'ensemble des villageois participe à cette fête et contribue à cet accueil, chacun à hauteur de ses moyens, offrant une volaille, un peu d'argent ou encore un pagne à la nouvelle venue. Cette organisation collective reste de mise ${ }^{24}$ mais elle semble s'appuyer sur des moyens de plus en plus importants. Lors de mariages récents, il a ainsi été fait état de dépenses substantielles pour mobiliser des groupes de griots musiciens réputés dans la région, mais aussi de surenchères dans les cadeaux faits à la fiancée par la famille de son futur époux et de partenaires extérieurs au village. La fête du mariage offre alors un espace de mise en scène ostentatoire, ce qui est une dimension inédite. Avant de conclure à un changement significatif, cette observation basée sur des cas particuliers mérite d'être confirmée par des enregistrements plus systématiques. Il reste qu'elle alimente d'ores et déjà la représentation d'une manifestation qui, en n'étant plus nécessaire à la mise en union, n'est plus non plus accessible à tous. On pourrait penser que cette évolution correspond à l'importation d'un modèle de cérémonie urbaine par le biais des migrations féminines. Il ne me semble pas que cela soit le cas: d'une part, en permettant aux jeunes filles de se constituer un trousseau par elles-mêmes, les migrations relativisent l'utilité de la cérémonie, favorisant plutôt un affaiblissement de cette pratique; d'autre part, on peut penser qu'une telle implantation serait apparue plus tôt, la pratique migratoire des hommes étant déjà ancienne. L'évolution dans le sens d'un surenchérissement et d'une privatisation de la fête du mariage traduit plus vraisemblablement, à mon sens, le recul d'une manifestation collective qui, en laissant ainsi libre un espace de mise en scène sociale, a permis sa réaffectation au bénéfice d'intérêts plus personnels. On y reviendra.

Si on considère que la formalisation des unions a notamment pour objectif d'assurer visibilité et promotion des compétences, des relations et des réseaux mobilisés, alors l'essor des unions «non fêtées $»^{25}$ apparaît comme une consécration du désengagement des instances collectives du champ matrimonial. Désengagement des acteurs lignagers qui, après s'être largement retirés du champ décisionnel et avoir consenti à l'assouplissement, en prestations et en durée, du processus matrimonial, paraissent désormais également démissionnaires de la gestion formelle. Désengagement de la communauté villageoise aussi qui n'est plus partie prenante dans l'accueil et l'installation du jeune couple. Avec la raréfaction de cette manifestation, on peut penser que ce sont à la fois la validation publique de la formation du couple et la fonction de la communauté villageoise (en tant que trame sociale différente de l'espace familial) dans l'intégration et l'identité de l'individu, qui sont mises en question.

24. Il ne semble pas y avoir une individualisation significative de la prise en charge des dépenses matrimoniales (prestations matrimoniales ou coût de la fête), qui repose généralement sur la famille du jeune homme, du moins les parents avec lesquels il travaille.

25. J'hésite à utiliser l'expression «d'union libre», car bien que leur union n'ait pas fait l'objet d'une cérémonie les officialisant, ces couples sont reconnus à part entière dès la cohabitation (ainsi on parle de l'épouse d'Untel ou du mari d'Untelle, qu'il y ait eu cérémonie ou non). 
Figure 2 Mariages (\%) ayant fait l'objet d'une fête au village, selon la génération de l'homme

Premiers mariages des hommes conclus avec une femme célibataire

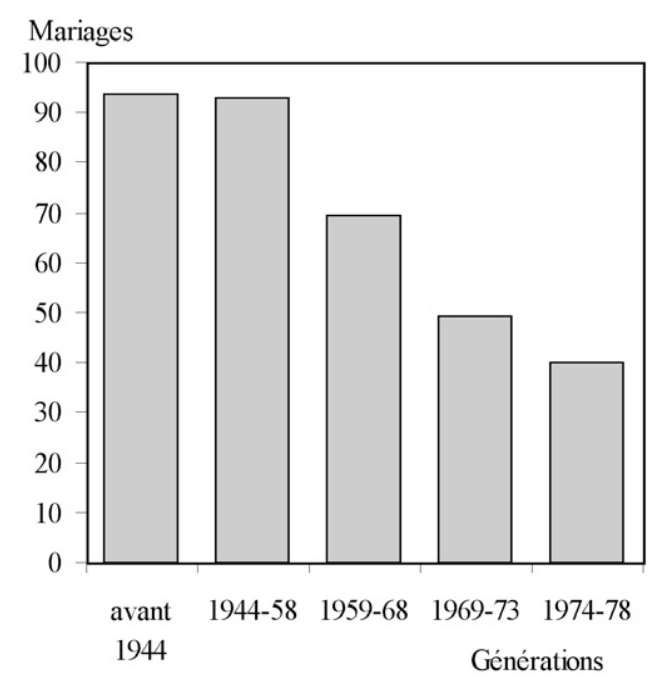

\section{INDIVIDUALISATION ET PRIVATISATION : QUELS ACTEURS ? QUELS RESSORTS?}

L'évolution des procédures matrimoniales rend compte d'une désagrégation progressive du dispositif institutionnel assurant l'encadrement de l'entrée en union des jeunes. Schématiquement, cette évolution peut être assimilée à la transition d'un régime de nuptialité contrôlée par les instances communautaires à un régime où les choix individuels prédominent. Pour autant, cette transition ne se résume pas à un mouvement continu, univoque, structuré par la confrontation de deux pôles homogènes d'intérêts opposés, d'ordre collectif ou individuel. Au contraire, la tendance observée se donne à lire comme une combinaison de temporalités et de forces variables. D'une part, la reconnaissance d'une place propre à l'individu dans la construction de son entrée en union n'est pas uniforme: l'individualisation ${ }^{26}$ des uns n'est pas forcément celle des autres. L'écart dans la temporalité et les modalités est net, par exemple, entre le développement d'une sphère d'intervention reconnue aux jeunes hommes et son équivalent féminin. D'autre part, les structures communautaires ne se laissent pas non plus réduire à un ensemble homogène. Les paliers de structuration (qu'ils relèvent du village ou de l'organisation familiale) sont susceptibles de porter des intérêts différents, particulièrement dans une conjoncture où les compétences sont en cours de redistribution. Cette géométrie variable (et dynamique) laisse penser que le conflit d'intérêts ne s'exprime pas nécessairement dans un binôme individu/communauté, se révélant plutôt en creux

26. Pour une discussion approfondie et particulièrement stimulante du concept d'individualisation, voir Alain Marie, 1997a, 1997b. 
dans le réagencement des unités collectives et l'affaiblissement des contrôles sociaux. Un mouvement de privatisation (comprise comme le glissement à une échelle familiale plus restreinte et plus concentrée de certains domaines de compétence et d'intervention) peut ainsi entretenir des tendances individualistes, et s'en nourrir, tout en ayant un pouvoir de subversion sur la charpente communautaire plus puissant que les comportements d'autonomie individuelle proprement dits.

En guise de conclusion, la dernière partie revient sur ces deux phénomènes d'individualisation et de privatisation, en s'interrogeant sur leur articulation avec les attentes individuelles d'une part, l'idéologie communautaire d'autre part.

\section{Implication individuelle et affaires collectives: des formes et des enjeux modulés par les rapports de genre}

Le relâchement de l'encadrement familial du mariage observé depuis 50 ans a été porté par des forces différentes. Deux périodes, deux contextes, deux logiques se distinguent: l'une couvrant approximativement les années 1970 et 1980, l'autre débutant avec les années 1990. La première correspond à une période de réaménagement dans l'organisation familiale et les rapports intergénérationnels, elle est associée à l'évolution statutaire des jeunes hommes. La seconde période se caractérise par des changements plus affirmés, moins contrôlés, elle est portée par un mouvement d'émancipation féminine.

Les années 1970-1980 correspondent à une période d'aménagement de l'économie familiale, avec d'un côté la diffusion de la charrue et de la culture attelée, de l'autre l'essor des migrations temporaires des jeunes hommes, destinées (notamment) à l'obtention de bêtes de labour. L'organisation matrimoniale s'assouplit dans le même temps, avec un accès facilité aux jeunes femmes (les jeunes hommes se marient plus jeunes, plus généralement avec une femme également célibataire), des procédures matrimoniales plus courtes et plus souples en termes de prestations, une participation du jeune homme au choix de son épouse. Il se construit ainsi un pôle de concertation à l'échelle familiale où les attentes masculines trouvent à s'exprimer, tandis que les autorités familiales continuent de prendre en charge la gestion matrimoniale. Aucun élément ne permet de penser que cette évolution ait été portée par un mouvement de revendication individuelle; au contraire, ces aménagements témoignent d'une souplesse qui assure la robustesse de l'institution matrimoniale.

Les années 1990 sont marquées par l'entrée des femmes dans la dynamique des changements sociaux locaux. Jusqu'alors réservées au sexe masculin, les migrations de jeunesse concernent également les jeunes femmes tout en revêtant des contours nouveaux. En effet, ces déplacements, systématiquement à destination de la ville, cessent vite d'être saisonniers pour se poursuivre sur plusieurs années, «vidant» des communautés villageoises de leurs groupes de jeunes filles. La pratique de la «jeunesse» qui était l'un des fondements de l'intégration et du contrôle social des jeunes générations se trouve ainsi minée dans les conditions mêmes de son exercice. Les migrations féminines se distinguent aussi de celles des jeunes hommes par leur absence d'articulation aux logiques familiales: souvent réalisées sans l'accord du responsable familial, elles répon- 
dent explicitement à des attentes individuelles et leur bénéfice, même modeste, n'est jamais versé au profit de l'économie domestique (Lesclingand, 2004a, 2004b). Les années 1990 sont marquées non seulement par l'essor des migrations féminines, mais aussi par une inflexion dans les tendances du mariage: l'âge d'entrée en union recule pour les deux sexes, le désengagement des instances familiales dans l'initiative de l'union s'accélère, la formalisation des unions, jusqu'alors préservée, lâche à son tour. Si ces changements ressemblent à un basculement de l'affaire matrimoniale des instances collectives aux mains des intéressé(e)s, peut-on pour autant les comprendre comme l'expression d'une appropriation par ces dernier(ère)s?

\section{Quels sont les acteurs des changements récents?}

Une association entre l'essor des migrations féminines et le relâchement des cadres matrimoniaux réguliers fait peu de doute tant les phénomènes sont concomitants ${ }^{27}$. Pourtant, si la migration est bel et bien revendiquée par les femmes comme une expérience personnelle, il est moins évident d'associer d'emblée l'essor des unions non formalisées à une appropriation féminine de l'entrée en union. Par exemple, on ne constate pas une augmentation des processus refusés par les femmes qui signifierait leur opposition croissante aux initiatives validées par leurs familles. Il n'apparaît pas non plus, dans les entretiens qualitatifs, de propos mentionnant l'intérêt d'une union non fêtée ${ }^{28}$; au contraire la fête du mariage reste perçue par les femmes comme un événement positif, gratifiant. Il semble bien que, concrètement, les responsables familiaux aient renoncé à leur intervention sans que celle-ci ne soit contestée ouvertement. Deux principaux facteurs ressortent des propos des villageois: d'une part le sentiment d'une perte de contrôle sur les jeunes, d'autre part le constat, qui s'impose de plus en plus avec la banalisation des unions réalisées en marge des cadres réguliers, d'une perte de nécessité. L’idée que le contrôle sur le mariage des filles était une bien difficile affaire a toujours existé, alimentée par les récits rares mais exemplaires de femmes ayant fui avec leur élu, laissant leur famille dans l'incapacité d'honorer leur engagement. Ce sentiment d'impuissance s'est encore renforcé avec les migrations féminines, l'apparition de grossesses préconjugales ${ }^{29}$ et la crainte que la jeune fille ne se marie en ville. Le

27. Pour une discussion plus détaillée sur l'articulation entre l'essor des migrations féminines et les changements matrimoniaux, voir Hertrich et Lesclingand (2007).

28. En revanche, le mariage légal est généralement perçu négativement par les femmes comme une perte d'autonomie. L'enregistrement d'un mariage légal permet en effet d'exiger le retour d'une épouse ayant quitté son conjoint et constitue ainsi une entrave au divorce.

29. Les enfants nés hors mariage prennent l'identité lignagère de leur mère et sont généralement élevés par leurs grands-parents maternels. Jusqu’aux années 1990, ces enfants (dénommés «enfants de rue») étaient rares; ils constituent aujourd'hui une catégorie de population visible dans les ménages de leurs grands-parents. L'identité problématique de ces naissances apparaît quand il est question de l'accès aux ressources et aux responsabilités lignagères des hommes: privés de filiation paternelle, ceux-ci ne sont pas insérés dans les trames lignagères le long desquelles s'organisent les transmissions. Gérables jusqu’à présent au cas par cas, ces situations, en devenant plus nombreuses, risquent de devenir un facteur de tension au sein des instances familiales et villageoises dans les prochaines décennies. 
désengagement des aînés s'est cependant manifesté avant que le phénomène ne devienne fréquent: parmi les jeunes femmes des générations 1970-1979 ayant réalisé une migration de travail avant leur mariage, $10 \%$ seulement se sont mariées sur le lieu de la migration. La banalisation du phénomène est par ailleurs un facteur qui a son importance: en se diffusant il gagne en légitimité et fournit la preuve que les exigences antérieures ont perdu leur nécessité.

Le pouvoir acquis par les femmes sur le mariage porte en fait d'abord sur le calendrier de leur entrée en union. Quand elles se sentent prêtes à se marier, les jeunes filles reviennent au village: une visite qui se prolonge au delà d'une semaine est un signe de disponibilité, très rapidement les jeunes femmes auront autour d'elles une cour de jeunes hommes, parmi lesquels elles pourront manifester leur préférence, notamment à l'occasion des causeries du soir. De fait, la plupart d'entre elles se marient dans les mois qui suivent leur retour au village (Lesclingand, 2004b). Il ne semble pas, du moins jusqu'à la fin des années 1990, que les femmes se soient approprié, sous forme réfléchie et anticipée, le choix de leur conjoint: la décision se fait rapidement parmi les candidats qui se manifestent, et non dans le cadre d'un projet conjugal qui aurait mûri entre les partenaires. L'implication des intéressés dans la décision matrimoniale ne semble donc pas signifier (ou pas encore) la construction d'un pôle d'autonomie conjugale.

\section{Privatisation et différenciation sociale}

J'ai souligné à plusieurs reprises que la démission croissante des instances lignagères du contrôle des unions n'était pas réductible à une question de conflit intergénérationnel. Certes les Anciens n'oublient pas les dissidences individuelles qui ont pu malmener leur autorité ou celle d'un autre, mais ces faits divers ressemblent parfois à de bien minces paravents... Si on approche le point du vue des individus au travers des récits qualitatifs, on identifie difficilement l'existence d'une contestation de l'autorité familiale en matière matrimoniale. Alors qu'ils trouvent à s'exprimer sur d'autres points (par exemple les conditions de vie pendant l'enfance), les sentiments d'injustice ou de frustration pouvant signifier la confrontation intergénérationnelle sont absents des récits que les hommes font de leur jeunesse et de leur entrée en union. L'intervention de la famille est jugée normale et légitime, quand elle n'est pas revendiquée: c'est le «travail» des responsables familiaux que de s'occuper du mariage des leurs et les jeunes hommes doivent pouvoir compter sur eux. Les femmes, comme on l'a vu, expriment plus souvent leur frustration de ne pas avoir été associées à la décision matrimoniale, mais sans pour autant formuler une contestation de l'autorité familiale. Le fait que les coûts matrimoniaux (prestations et fête) restent pris en charge par la famille, sans incomber au marié personnellement, est également significatif de l'absence de conflit ou de contournement individuel de prérogatives familiales.

Si les responsables familiaux n’ont pas été acculés par les intéressés à se retirer du champ matrimonial et s'ils gagnent visibilité et légitimité dans l'exercice de ces compétences, alors pourquoi y renoncent-ils? C'est peut-être dans les tensions internes aux institutions familiales qu'il faut en chercher la raison. Bien que ce facteur ne soit 
jamais mis en avant et que la cohésion de l'unité lignagère reste une valeur affichée par tous, différents éléments ${ }^{30}$ montrent que les lignages sont traversés par des dissensions croissantes. Les problèmes fonciers en sont une expression, dont témoigne le recours plus fréquent aux instances administratives en cas de litige, ou encore les difficultés exprimées par certains émigrés revenus au village pour disposer de bonnes terres. Outre la gestion foncière, d'autres compétences reconnues aux lignages perdent en importance, en efficacité ou en visibilité. Ainsi en est-il des compétences religieuses (cultes villageois et lignagers, initiation des enfants) dont la dimension communautaire devient moins évidente avec l'hétérogénéité introduite par le christianisme. L'évolution des activités et de l'identité villageoise passe désormais par des canaux non traditionnels: école, développement rural, action sanitaire, démocratisation politique... Ceci motive la création d'autres axes de structuration, particulièrement dans le contexte actuel de décentralisation. L'importance des migrations contribue, sur un autre plan, à la construction de relations familiales plus personnalisées, ne serait-ce que parce que l'envoi d'un courrier nécessite la désignation d'un destinataire... Les conditions favorables à la création de communautés d'intérêts autonomes à l'échelle intrafamiliale existent à différents niveaux. Cette tendance apparaît aussi dans le domaine matrimonial. Nos données ne permettent pas d'aborder la question de manière très détaillée: il faudrait pour cela pouvoir retracer le circuit des paroles échangées autour d'un mariage au sein du lignage. Cependant, deux indicateurs formels suggèrent une prise de distance par rapport à l'ordre lignager: la raréfaction des unions initiées par la parenté éloignée et la quasi-disparition du recours à un membre du lignage comme médiateur de l'affaire matrimoniale.

L'affaiblissement de l'encadrement formel des unions peut être compris en rapport avec cette dynamique de recomposition familiale, à la fois comme cause et comme conséquence: d'une part la perte de cohésion lignagère amoindrit la demande de formalisation et de visibilité lignagère, d'autre part la simplification des démarches permet leur mise en œuvre à l'échelle désagrégée. Dans le contexte actuel, un père de famille peut espérer gérer le mariage de ses enfants de façon simplifiée, en s'en tenant à une information de principe du responsable lignager, sans avoir à mobiliser des réunions lignagères, sans avoir à tenir compte de l'avis de ses aînés ni à solliciter leurs ressources matérielles et symboliques... L'émancipation des cadres matrimoniaux ne se comprend donc pas seulement (ni peut-être principalement) par rapport aux intéressés, mais aussi par rapport à leurs proches parents.

Cette privatisation est en elle-même un facteur de remise en cause des forces d'homogénéisation communautaires. Dès lors que les pères gèrent directement les unions de leurs enfants et que les formalités ne sont plus standardisées, ni nécessaires, il devient possible d'utiliser et d'instrumentaliser le mariage à des fins privées. On en observe les premiers signes aujourd'hui dans les nouvelles formes que revêt la fête du mariage.

30. Les réflexions développées dans cette partie s'appuient principalement sur des observations de terrain; elles sont de nature plus spéculative que les analyses précédentes, basées sur des données statistiques. 
Tant qu'elle était systématique, cette fête était réalisée avec les moyens du bord, avec la vocation de réunir dans un moment de convivialité la communauté villageoise autour de la nouvelle épouse. Aujourd'hui plus rare, elle mobilise plus de moyens et prend des formes ostentatoires inédites. La distinction qu'elle introduit se perçoit aussi dans le recours à de nouveaux modes de communication, comme les communiqués radio ou les cartons d'invitation, dans une population où l'accès à l'écrit reste pourtant marginal. La fête du mariage devient un espace de différenciation sociale, qui imprime ses marques à la fois au niveau des organisateurs et du public: le nouveau standard, s'il se confirme, ne permettra plus à chacun d'organiser une fête du mariage, il ne permettra plus non plus (compte tenu de la valeur des cadeaux affichés) à chacun d'y participer.

Finalement tout laisse à penser que le mariage est amené à rester un élément important du jeu collectif, mais avec un glissement de paradigme: il était une composante du contrôle intergénérationnel, il devient un instrument de différenciation sociale.

\section{An nexe L'enquête biographiQue. Données Collectées, données ANALYSÉES}

\section{Population enquêtée, effectifs traités}

L'enquête initiale a été réalisée sur le mode rétrospectif en 1988-1989 puis actualisée à trois reprises (1994-1995, 1999-2000, 2004). À chacun des passages, les biographies déjà enregistrées ont été actualisées et celles des nouveaux venus ont été recueillies complètement. Les biographies ont été mises à jour jusqu'à la date de l'enquête pour les résidants et les hommes partis en migration (l'enregistrement étant réalisé auprès des parents résidant au village). Les femmes parties en migration ont également été suivies tant qu'elles étaient célibataires, la migration liée au mariage correspondant à une sortie d'observation.

Dans cet article, les analyses sont réalisées sur les biographies des individus interrogés au titre de résidant à l'un au moins des passages de l'enquête. L'actualisation de la biographie matrimoniale est prise en compte jusqu'à la limite prévue au suivi (le dernier passage de l'enquête pour les hommes émigrés, la migration matrimoniale pour les femmes émigrées, ou encore le décès) même si l'individu ne réside pas au village à cette échéance. L'intérêt de la démarche est d'éviter les biais d'analyse dus au caractère sélectif de l'émigration sur les célibataires. En effet, sachant que l'émigration est plus forte parmi les célibataires, une analyse limitée aux seules résidants conduirait à sous-estimer l'âge au mariage parmi les jeunes générations. L'effectif d'enquêtés concernés est de 1310 (604 hommes, 706 femmes), en se limitant aux générations nées avant 1984 .

\section{Les informations collectées dans la biographie matrimoniale}

Initialement conçu sous la forme classique d'un enregistrement événementiel, le questionnaire biographique a été enrichi à mi-parcours de l'enquête initiale pour saisir 
l'évolution des contrôles familiaux sur les événements individuels. Le module initial saisissait les unions successives, leur issue et un certain nombre de caractéristiques des deux conjoints. Dans la version remaniée, le questionnaire enregistre non seulement les unions, mais aussi les procédures matrimoniales qui ont été rompues avant d'aboutir au mariage. Ce sont ainsi les mariages potentiels ou projets d'union qui ont été enregistrés. Pour chaque mariage ou processus matrimonial ${ }^{31}$, des questions précises ont été posées sur les différentes étapes:

- La décision de l'union: initiative de l'union et recueil de l'accord formel de la famille de la fille, qui marque formellement le début d'une procédure traditionnelle;

- Les prestations engagées, de type traditionnel (travaux agricoles, remise de grains) et moderne (remise directe d'argent ou achat de bière de mil);

- La période transitoire, dite de déposition, qui précède le mariage et pendant laquelle la fille est confiée à une famille désignée par celle de son futur époux;

- La durée du processus;

- La sanction sociale de l'union: fête du mariage au village, mariage légal, mariage religieux.

Les procédures matrimoniales sont analysées en privilégiant les biographies matrimoniales collectées auprès des hommes ${ }^{32}$, et en se limitant aux générations nées avant 1979, qui pour la plupart ont intégralement vécu la période d'entrée en union avant le dernier passage de l'enquête ${ }^{33}$. Les traitements relatifs aux procédures matrimoniales portent sur 241 observations, correspondant aux premiers mariages des hommes nés avant 1979, conclus avec une femme également célibataire et dont le détail des procédures matrimoniales a été enregistré ${ }^{34}$.

31. Ces questions ne concernent que les démarches engagées pour un mariage avec une femme célibataire, les remariages féminins ne faisant pas l'objet de procédures matrimoniales.

32. Les femmes semblent avoir une connaissance plus partielle des procédures engagées pour leur mariage que les hommes, en particulier au sein des anciennes générations (Hertrich, 1997).

33. Cette restriction est nécessaire à la comparabilité des pratiques entre générations: si l’on prend en compte les mariages des générations en âge de se marier, on sélectionne des hommes qui, en moyenne, sont entrés en union plus jeunes que l'ensemble de leur génération, dont on peut penser qu'ils se distinguent également par les modalités des procédures matrimoniales. Dans la population étudiée, la quasi-totalité (plus de $95 \%$ ) des premiers mariages masculins ont lieu avant 29 ans: l'entrée en union des générations nées avant 1974 (30 ans au dernier passage, en 2004) peut donc être suivie intégralement. L'observation a été élargie aux générations 1974-1978, bien que $23 \%$ des hommes y soient encore célibataires: d'une part pour être en mesure de suivre et conforter les changements engagés à partir du groupe de générations précédentes, d'autre part car il me semble probable que le biais introduit va dans le sens d'une sous-estimation des changements en cours (il est probable que les hommes se distinguant par un célibat prolongé se distinguent également par un moindre encadrement communautaire) et ne remet donc pas en cause le sens de l'évolution.

34. On peut résumer comme suit les transitions entre l'effectif des générations traitées (nées avant 1979) et celui des mariages analysés: sur 464 hommes enquêtés, 415 sont non-célibataires, parmi ceux-ci 339 ont conclu leur premier mariage avec une femme célibataire et le détail de la procédure matrimoniale a été saisi pour 241 (soit $71 \%$ ) de ces mariages. 


\section{RÉSUMÉ}

L'article examine l'individualisation des formes d'entrée en union dans une population rurale du Mali, à partir d'une enquête biographique détaillant les procédures matrimoniales (initiative, prestations, médiatisation et formalisation) et à partir d'entretiens qualitatifs. II relativise l'idée d'un mouvement continu et homogène bâti sur la contestation individuelle des contrôles familiaux. Le mariage a d'abord évolué par réajustements successifs en intégrant les attentes masculines au dispositif formel géré par les aînés. Il connaît une évolution récente plus brutale, marquée par le recul de la formalisation des unions et le désengagement des responsables familiaux de la prospection matrimoniale. Ce tournant est associé à l'essor des migrations féminines qui met à mal le dispositif institutionnel d'encadrement des jeunes. L'individualisation se double d'un mouvement de privatisation des affaires matrimoniales. Le mariage reste un élément important du jeu collectif, mais avec un glissement de paradigme: il était une composante du contrôle intergénérationnel, il devient aujourd'hui un instrument de différenciation sociale.

\section{ABSTRACT}

This article examines the various ways in which unions are organized in the rural population in Mali using data from a biographical survey exploring matrimonial procedures (initiative, services rendered, mediatization and formalization) and from a series of qualitative interviews. It renders more relative the notion there is a continual, homogenous movement based on the individual contesting family control. Marriage has evolved firstly through the successive readjustments integrating masculine expectations into the formal framework managed by the elders. There has recently been a more brutal change showing the decline of formal unions and that of family responsibility in matrimonial prospecting. This evolution is related to the increase of female migration undermining the institutional control over young people. A privatizing movement of matrimonial affairs reinforces individualization. Marriage remains an important element in the collective game but slides towards paradigm; it was a component of inter-generational control, it is now an instrument for social differentiation.

\section{RESUMEN}

El artículo examina la individualización de las formas de unión en una población rural de Malí, a partir de una investigación biográfica detallando los procedimientos matrimoniales (iniciativa, prestaciones, mediatización y formalización) y a partir de entrevistas cualitativas. Relativiza la idea de un movimiento continuo y homogéneo construido sobre la oposición individual de los controles familiares. El matrimonio en primer lugar ha evolucionado por reajustes sucesivos integrando las expectativas masculinas al dispositivo formal administrado por los mayores. Conoce una reciente evolución más brutal, caracterizada por el retroceso de la formalización de las uniones y el desinterés de los responsables familiares de la prospección matrimonial. Este cambio de dirección está asociado al crecimiento de las migraciones femeninas que disminuye el dispositivo institucional de control de los jóvenes. La individualización se duplica de un movimiento de privatización de los asuntos matrimoniales. El matrimonio sigue siendo un elemento importante del funcionamiento colectivo, pero con un deslizamiento de paradigma: era un componente del control intergeneracional, que se convierte hoy en un instrumento de diferenciación social. 


\section{BIBLIOGRAPHIE}

Antoine, P. (2002). «Les complexités de la nuptialité: de la précocité des unions féminines à la polygamie masculine en Afrique», in G. Caselli, J. Vallin et G. Wunsch (dir.), Démographie et synthèse. II. Les déterminants de la fécondité, Paris, INED/PUF, p. 75-102.

Antoine, P., M. Razafindrakoto et F. Roubaud (2001). «Contraints de rester jeune? Évolution de l'insertion dans trois capitales africaines: Dakar, Yaoundé, Antananarivo ", Autrepart, n 18, p. 17-36.

Bledsoe, C. et G. Pison (éd.) (1994). Nuptiality in Sub-Saharan Africa. Contemporary Anthropological and Demographic Perspectives, Oxford, Clarendon Press.

Burnham, P. (1987). «Changing Themes in the Analysis of African Marriage», in D. Parkin et D. Nyamwaya (éds.), Transformations of African Marriage, Londres, International African Seminars, p. 37-54.

Calvès, A.-E. (2007). «Trop pauvre pour se marier? Crise de l'emploi urbain et entrée en premère union des hommes au Burkina Faso ", Population, no 62, p. 339-360.

Capron, J. (1988a). Septétudes d'ethnologie bwa: Mali-Burkina Faso. 1957-1987, Tours, Université FrançoisRabelais, Mémoire du Laboratoire d'Anthropologie et de Sociologie, $\mathrm{n}^{\circ} \mathrm{I}$.

Capron, J. (1988b). Introduction à l'étude d'une société villageoise. 1955-1968, Tours, Université FrançoisRabelais, Mémoire du Laboratoire d'Anthropologie et de Sociologie, $\mathrm{n}^{\circ}$ II.

Capron, J. (1973). Communautés villageoises bwa: Mali-Haute Volta, Paris, Museum National d'Histoire Naturelle.

Comaroff, J. (1980). «Introduction», in J. Comaroff (éd.), The Meanings of Marriage Payments, London, Academic Press, p. 1-47.

Diarra, J. (1994). Les Bwa (Mali-Burkina Faso) dans l'historiographie: essai d'analyse critique et interprétation d'un processus de marginalisation, Paris, Centre de Recherches Africaines de l'Université de Paris, Institut Catholique de Paris, Mémoire de DEA.

Enel, C., G. Pison et M. Lefebvre (1994). «Migration and Marriage Change: A Case Study of Mlomp, a Joola Village in Southern Senegal », in C. Bledsoe et G. Pison (éd.), Nuptiality in Sub-Saharan Africa: Contemporary Anthropological and Demographic Perspectives, Oxford, Clarendon Press, p. 92-116.

Guigou, B. (1992). Les changements du système familial et matrimonial: les Sérères Sine (Sénégal), Paris, École des Hautes Études en Sciences Sociales, Thèse de doctorat.

GuYer, J. (1981). «Household and Community in African Studies», African Studies Review, vol. 24, n 2-3, p. 87-137.

Hertrich, V. (2008) (à paraître). «La polygamie: persistance ou recomposition? Le cas d'une population rurale du Mali», Cahiers québecois de démographie.

Hertrich, V. (2007). «Nuptialité et rapports de genre en Afrique: tendances de l'entrée en union, 1950-99», in T. LocoH (dir.), Genre et société en Afrique, Paris, INED, p. 281-307.

Hertrich, V. (1997). «Les réponses des hommes valent-elles celles des femmes? Une double collecte sur les questions génésiques et matrimoniales dans une population du Mali», Population, n 1, p. 45-62.

Hertrich, V. (1996). Permanences et changements de l'Afrique rurale: dynamiques familiales chez les Bwa du Mali, Paris, CEPED.

Hertrich, V. et M. Lesclingand (2007). Transition to Adulthood and Gender: Changes in Rural Mali, Paris, INED.

Hertrich, V. et M. Lesclingand (2003). «Jeunesse et passage à l'âge adulte chez les Bwa du Mali, in V. Hertrich et S. KeÏTA (éd.), Questions de population au Mali, Bamako (Mali), Le Figuier, UNFPAMali, p. 251-281.

Hertrich, V. et T. Locoh (1999). Rapports de genre, formation et dissolution des unions dans les pays en développement, Liège, UIESP.

Isiugo-Abanine, U. (1995). «Bridewealth, Marriage and Fertility in the East-Central States of Nigeria, Genus, vol. 61, n 3-4, p. 151-178. 
Isiugo-Abanihe, U. (1994). «Consequences of Bridewealth Changes on Nuptiality Patterns Among the Ibo of Nigeria», in C. Bledsoe et G. Pison (éd.), Nuptiality in Sub-Saharan Africa: Contemporary Anthropological and Demographic Perspectives, Oxford, Clarendon Press, p. 74-91.

Keïta S. et F. O. Konaté (2003). «Le Mali et sa population», in V. Hertrich et S. KeÏta (éd.), Questions de population au Mali, Bamako, Le Figuier, UNFPA-Mali, p. 11-48.

Lallemand, S. (1977). Une famille mossi, Paris/Ouagadougou, Recherches voltaïques, CNRS/CVRS.

LEGUY, C. (2001). Le proverbe chez les Bwa du Mali: parole africaine en situation d'énonciation, Paris, Karthala.

Lesclingand, M. (2004a). «Nouvelles stratégies migratoires des jeunes femmes rurales au Mali: de la valorisation individuelle à une reconnaissance sociale», Sociétés contemporaines, ${ }^{\circ} 55$, p. 21-42.

Lesclingand, M. (2004b). Nouvelles pratiques migratoires féminines et redéfinition des systèmes de genre: une analyse à partir des changements démographiques en milieu rural malien, Paris, Institut d'études politiques, Thèse de doctorat.

Lesclingand, M. et V. Hertrich (2006). «Family Structure and Labor Migration: A Longitudinal Study in Rural Mali», Communication à l'European Population Conference (Liverpool, 21-24 juin 2006).

LLoyd, C. B. (2005). «The Transition to Marriage», in C. B. LLoyd (éd.), Growing Up Global: The Changing Transitions to Adulthood in Developing Countries, Washington, National Academies Press, p. 416-505.

Locoн, T. (1994). «Social Change and Marriage Arrangements: New Types of Union in Lomé, Togo», in C. Bledsoe et G. Pison (éd.), Nuptiality in Sub-Saharan Africa: Contemporary Anthropological and Demographic Perspectives, Oxford, Clarendon Press, p. 215-230.

MaIr, L. (1974). Le mariage: étude anthropologique, Paris, Payot.

Marcoux, R. et V. Piché (1998). «Crise, pauvreté et nuptialité à Bamako», in F. Gendreau (dir.), Crise, pauvreté et changements démographiques dans les pays $d u$ Sud, Paris, AUPELF-UREF/ESTEM, p. 237255.

MARIE, A. (1997a). «Du sujet communautaire au sujet individuel: une lecture anthropologique de la réalité africaine contemporaine, in A. MARIE (éd.), L'Afrique des individus: itinéraires citadins dans l'Afrique contemporaine (Abidjan, Bamako, Dakar, Niamey), Paris, Karthala, p. 53-110.

Marie, A. (1997b). «Individualisation: entre communauté et société, l'avènement du sujet», in A. Marie (éd.), L'Afrique des individus: itinéraires citadins dans l'Afrique contemporaine (Abidjan, Bamako, Dakar, Niamey), Paris, Karthala, p. 407-436.

Nagashima, N. (1987). "Aspects of Change in Bridewealth Among the Iteso of Kenya», in D. PArkin et D. Nyamwaya (éd.), Transformations of African Marriage, Londres, International African Seminars, p. 183-198.

Parkin D. et D. Nyamwaya (1987). «Introduction: Transformations of African Marriage, Change and Choice », in D. PARkin et D. Nyamwaya (éd.), Transformations of African Marriage, Londres, International African Seminars, p. 1-16.

République du Mali (2001). Recensement général de la population et de l'habitat, avril 1998: résultats définitifs. Vol. 1. Série socio-démographique, Bamako, DNSI.

Rondeau, C. (1994). Les paysannes du Mali: espaces de liberté et changements, Paris, Karthala.

SAvonnet-Guyot, C. (1986). État et sociétés au Burkina: essai sur le politique africain, Paris, Karthala.

TAвет, P. (1985). «Fertilité naturelle, reproduction forcée», in N.-C. MAтHieu (éd.), L'arraisonnement des femmes: essais en anthropologie des sexes, Paris, Éditions de l'École des Hautes Études en Sciences Sociales, p. 61-146. 